\title{
Estimating Emissions from Static Traffic Models: Problems and Solutions
}

\author{
Nikolaos Tsanakas $\mathbb{D}^{1},{ }^{1}$ Joakim Ekström $\mathbb{D}^{1},{ }^{1}$ and Johan Olstam $\mathbb{D}^{1,2}$ \\ ${ }^{1}$ Communications and Transport Systems, Department of Science and Technology, Linköping University, \\ Norrköping SE-601 74, Sweden \\ ${ }^{2}$ The Swedish National Road and Transport Research Institute, Linköping SE-581 95, Sweden \\ Correspondence should be addressed to Nikolaos Tsanakas; nikolaos.tsanakas@liu.se
}

Received 19 June 2019; Accepted 19 December 2019; Published 1 February 2020

Academic Editor: Jose E. Naranjo

Copyright (C) 2020 Nikolaos Tsanakas et al. This is an open access article distributed under the Creative Commons Attribution License, which permits unrestricted use, distribution, and reproduction in any medium, provided the original work is properly cited.

\begin{abstract}
In large urban areas, the estimation of vehicular traffic emissions is commonly based on the outputs of transport planning models, such as Static Traffic Assignment (STA) models. However, such models, being used in a strategic context, imply some important simplifications regarding the variation of traffic conditions, and their outputs are heavily aggregated in time. In addition, dynamic traffic flow phenomena, such as queue spillback, cannot be captured, leading to inaccurate modelling of congestion. As congestion is strongly correlated with increased emission rates, using STA may lead to unreliable emission estimations. The first objective of this paper is to identify the errors that STA models introduce into an emission estimation. Then, considering the type and the nature of the errors, our aim is to suggest potential solutions. According to our findings, the main errors are related to STA inability of accurately modelling the level and the location of congestion. For this reason, we suggest and evaluate the postprocessing of STA outputs through quasidynamic network loading. Then, we evaluate our suggested approach using the HBEFA emission factors and a $19 \mathrm{~km}$ long motorway segment in Stockholm as a case study. Although, in terms of total emissions, the differences compared to the simple static case are not so vital, the postprocessor performs better regarding the spatial distribution of emissions. Considering the location-specific effects of traffic emissions, the latter may lead to substantial improvements in applications of emission modelling such as dispersion, air quality, and exposure modelling.
\end{abstract}

\section{Introduction}

The traffic situation in urban areas around the world is today characterised by severe road congestion. Congestion increases travel times, but usually also results in increased energy usage and vehicular emissions. Exhaust emissions from road traffic is an important contributor to the main air pollutants, with significant effects on the urban air quality [1]. A more efficient usage of the transportation systems is therefore of great importance. By taking the correct decisions and applying the appropriate measures, transportation planners and policy makers can alleviate congestion and hence reduce its negative impacts. Traffic models are useful tools for the evaluation of changes in a traffic system. In the case of analysing the impact on the environment or energy use, traffic models are coupled with emission models. In this integrated modelling framework, traffic models generate the required emission modelling inputs, i.e., traffic state and traffic activity, while emission models estimate energy use and the amount of pollutant emitted per vehicle and distance.

In large urban areas, traffic data used for exhaust emission and air pollution analyses are commonly derived by traffic assignment models. Traffic assignment models are used for transport planning and economic appraisals, and their aim is to generate traffic volumes on road segments, relying on specific behavioural principles [2]. They intrinsically describe the interaction between travel demand infrastructure supply employing two submodels, the route choice and the network loading models. A fundamental 
classification of traffic assignment models concerns the temporal dimension, categorising the models either as static or dynamic [3].

Despite the extensive research on Dynamic Traffic Assignment (DTA) during the last decades, Static Traffic Assignment (STA) is still the most commonly applied approach for analysing emissions or other externalities of traffic. STA models are used for the strategic assessment of the current or future state of a transportation network assuming a stationary travel demand and infrastructure supply. These types of models have been proposed over 50 years ago, and their attributes of efficiency, accountability, and robustness are well studied and widely accepted. Therefore, STA models have been favoured by policy makers due to their ease of use, reduced computational cost, and low requirements of input data.

However, several limitations of STA have also been identified and discussed in many studies [4-8]. The underlying assumptions of STA, related to the stationary demand, long aggregation intervals, and static network loading, can lead to unrealistic results when variations in traffic conditions are high. Network loading is instantaneous, and thus many important dynamic traffic flow phenomena, such as propagation of queues and spillback, cannot be taken into account. This can result in an inaccurate estimation of dynamic traffic variables, such as the level and location of congestion, affecting emission estimation analyses. Congestion is strongly correlated with increased emission rates as it is associated with low speeds and stop and go conditions. More specifically, in the case of a bottleneck, all the time delays, and consequently the high emission rates, are assigned by STA models at the bottleneck link. The links upstream the bottleneck remain unaffected. However, in reality, increased delays and emissions are observed upstream the bottleneck due to the queue spillback. Moreover, STA models may overestimate the emissions downstream the bottleneck being unable to capture flow metering effects.

STA models are still used for the purposes of strategic transport planning relying on the hypothesis that accumulated delays at the bottleneck could compensate the delays, observed in reality elsewhere in the network. In the cases where planning and policy making is based on travel times, the accurate congestion's location may not be so crucial, since the total origin-destination travel times could be accurately modelled by a well-calibrated STA model. However, the accurate determination of the level and the location of congestion is essential from an environmental analysis perspective, since several applications of emission modelling have location-specific effects.

Applications of emission modelling, such as dispersion and exposure modelling, are sensitive to the spatial distribution of emission rates. Dispersion models consider location-specific background pollution and meteorology data. In addition, different network links are associated with different number of pedestrians, cyclists, and people living or working by the roadside and are exposed to emissions. This could have a direct effect on an economic analysis, such as a cost-benefit analysis, where the monetary cost of a gram of pollutant emitted is analogous to the number of people exposed and affected [9]. Accordingly, in such an analysis, the travel time costs are associated with route travel times (from origin to the destination) and the use of STA may be sufficient. In most of the cases, though, STA models are used for both travel time and emission estimation.

While STA models consider a single time period and the traffic variables are expressed as averages over this period, DTA models consider several shorter time periods. Additionally, in contrast to STA models, DTA can model spillback of the queues as traffic demand exceeds capacity and dispersion of the queues when demand is below the capacity. Trying to take advantage of the dynamic modelling benefits and potentially improve emission estimation, Aziz and Ukkusuri [10], Smit et al. [11], Aguilera and Lebacque [12], Borge et al. [13], and Zhou et al. [14] applied dynamic traffic models in order to generate inputs for emission models. A review of the DTA models used for environmental analyses can be found in Wismans et al. [8], while Wang et al. [7] highlighted the methodological advantages of DTA in emission modelling. Furthermore, in Wismans et al. [15], a comparison between the emission estimations based on static and dynamic traffic models is performed, resulting in large absolute differences between the two approaches.

Although DTA approaches are acknowledged to be more capable of modelling traffic congestion, their performance heavily depends on the availability and the quality of the input data. Complexity issues make dynamic modelling computationally expensive and time consuming to calibrate, discouraging their application in larger areas. In addition, DTA models do not possess the property of unique link flows, which is an important feature when using model output for policy or project evaluations.

A middle-ground solution between static and dynamic modelling could be the postprocessing of static models using quasidynamic network loading approaches, such as the ones described by Bliemer et al. [16] and Bundschuh et al. [17]. These models can be placed in the middle of STA and DTA both in terms of computing efficiency and reliability. Quasidynamic loading incorporates queue spillback and flow metering effects by setting maximum density and capacity constraints, respectively. Therefore, such approaches may lead to a more realistic location of congestion, which potentially can improve the accuracy of emission estimations.

This paper aims to investigate the errors that static modelling outputs introduce in the emission estimation process and to suggest and evaluate improvements in the modelling process. Preliminary results from this study have earlier been presented at the Swedish transportation research conference [18]. The paper extends Tsanakas et al. [19] with methodological development and an extended case study and is structured as follows. Section 2 provides a description of macroscopic emission models and their use with static traffic models, together with an overview of developed methods for postprocessing static model data. Section 3 describes the different sources of emission estimation errors that static models can result in, with illustrative examples for a motorway stretch in Stockholm. In Section 4, a quasidynamic approach for postprocessing the emission estimates obtained from static models is described. 
This approach is then evaluated for the same motorway stretch in Stockholm. Finally, Section 5 provides some conclusions and suggestions for further research.

\section{Macroscopic Emission Models with Static Traffic Data}

2.1. Macroscopic Emission Modelling. Emission factors and traffic activity are the basis of each macroscopic emission model. Emission factors provide the amount of emitted pollutants in relation to traffic activity, as a function of the traffic state. Commonly, emission factors are given in terms of grams of pollutant emitted per vehicle kilometre for each vehicle and road category, while traffic activity is provided as traffic flow in number of vehicles per time unit. The values of emission factors are usually obtained from detailed vehicle simulation of specific driving cycles. These driving cycles represent real-world driving for the specified vehicle and road category. A detailed review of approaches used for deriving emission factors is given by Franco et al. [20].

In Europe, HBEFA and COPERT are the two mainly used macroscopic emission models. The two models are based on the same model for microsimulation of emissions, PHEM (Passenger Car and Heavy Duty Emission Model). However, they differ in how the functional relationship between the traffic state and emission factors is formulated and in the development of the underlying driving cycles fed to PHEM. For a detailed description of the two models, we refer to Franco et al. [21].

HBEFA (Handbook on Emission Factors for Road Transport) [22] was developed by Germany, Austria, Switzerland, Norway, and Sweden and can be used for estimating emissions on single roads, as well as on complete traffic systems at regional and national level. HBEFA is essentially a database which includes a large number of emission factors corresponding to different vehicle classes, road categories, and traffic situations. The traffic situations are divided into the four classes: Free flow, Heavy, Saturated, and Stop and go. Road types are categorised by their road environment (rural or urban) and their speed limit and road type (Motorway, Trunk road/Primary, Distributor/Secondary, Local/Collector, and Access/Residential). For each traffic situation, the emission factor is constant, and each traffic situation is qualitatively defined by a characterisation of the traffic situation. In practice, this qualitative description needs to be quantified, which is usually done by formulating thresholds based on mean speed and/or traffic flow. Thus, the emission factors can be viewed as discrete functions of speed and/or flow.

COPERT (Computer programme to calculate emissions from road transport) [23] has been adopted by several European countries to officially report their national inventories of emissions from road transport. COPERT is also the main emission model used in the EMEP/EEA Atmospheric Emissions Inventory Guidebook [24]. Similar to HBEFA, COPERT emission factors depend on vehicle class and road type, but in contrast to HBEFA, the emission factors are given as continuous functions of average speed.

For this study, HBEFA emission factors have been used for estimating the emissions of five pollutants, $\mathrm{HC}, \mathrm{CO}$,
$\mathrm{NO}_{\mathrm{x}}, \mathrm{CO}_{2}$, and $\mathrm{PM}_{10}$. Our study includes two different road types, urban motorway speed limit $70 \mathrm{~km} / \mathrm{h}$ and urban motorway with speed limit $90 \mathrm{~km} / \mathrm{h}$. The emission factors are computed by weighting the emission factors of 15 different vehicle classes of the Swedish vehicle fleet composition (see Table 2 in Appendix A), both in terms of fleet mix and mileage (only petrol, diesel, and alternative fuel cars are included in this study). Figure 1 illustrates the HBEFA emission factors for $\mathrm{CO}$ and $\mathrm{NO}_{\mathrm{x}}$, corresponding to the Swedish vehicle mix, while Table 3 in Appendix B contains the full set of emission factors used in this study. We can notice that for some pollutants, free flow conditions may lead to higher emission factors compared to the corresponding ones produced by heavy traffic conditions. In such cases, a slight increase in congestion will possibly lead to reduced emission rates.

\subsection{Determining Traffic Situations Based on Static Traffic} Data. The traffic situation as well as the traffic activity has to be determined first in order to apply the HBEFA emission factors. The traffic situation is usually determined by traffic analysis tools such as STA models. When an analysis is based on an STA model, the traffic network is represented as a directed graph, $\mathscr{G}=(\mathscr{N}, \mathscr{A})$, which includes a set of nodes, $\mathcal{N}$, and a set of links, $\mathscr{A}$, where each link is associated with different attributes, such as capacity, $C_{a}$, and length, $L_{a}$. Let $\mathscr{R}$ be the set of origin nodes associated with the origin zones, $\mathscr{R} \subseteq \mathcal{N}$, and $\mathcal{S}$ be the set of destination nodes concerning the destination zones, $\mathcal{S} \subseteq \mathcal{N}$. The desired number of movements from an origin node, $r \in \mathscr{R}$, to a destination node, $s \in \mathcal{S}$, is denoted by $q_{r s}$, and is expressed by an origindestination (O-D) matrix. Furthermore, let $\mathscr{K}^{r s}$ be the set of different alternative link sequences, called routes or paths that connect each origin node, $r \in \mathscr{R}$, with a destination node, $s \in \mathcal{S}$. The problem of traffic assignment, then, concerns how the demand, $q^{r s}$, between each origin-destination pair, $r s$, will be distributed among the possible paths $k, k \in \mathscr{K}^{r s}$, given that link travel time, $t_{a}$, is a function of link flow, $Q_{a}$, for each $a \in \mathscr{A}$ [2].

The assignment usually relies on behavioural principles, such as the User Equilibrium (UE) [25]. UE is a stable condition where all the available paths $k, k \in \mathscr{K}^{r s}$, have the same travel time and no traveller can improve his or her travel time by unilaterally changing routes. To find the equilibrium solution in a network, STA problem was mathematically formulated as an optimisation problem ((1a)-(1d)), by Beckmann et al. [26]:

$$
\min z(x)=\sum_{a \in \mathscr{A}} \int_{0}^{Q_{a}} t_{a}(\omega) \mathrm{d}(\omega),
$$

subject to

$$
\begin{aligned}
\sum_{k \in \mathscr{K}^{r s}} f_{k}^{r s} & =q_{r s}, \quad \forall r, s, \\
f_{k}^{r s} & \geq 0, \quad \forall k, r, s,
\end{aligned}
$$

and the definitional constraint 


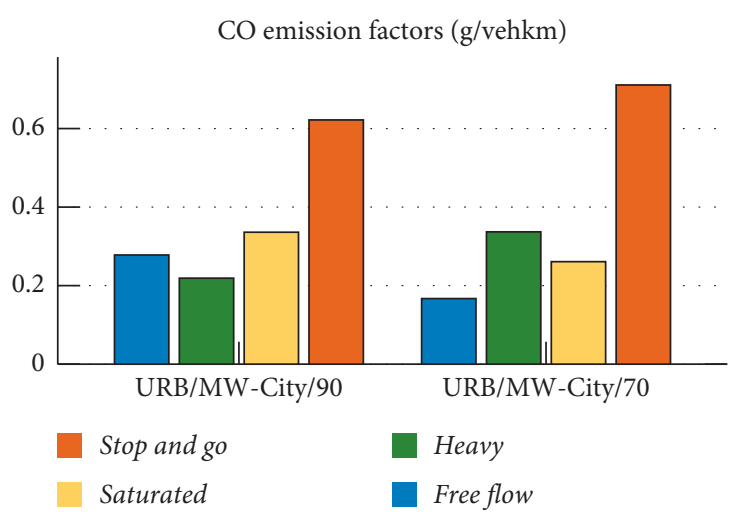

(a)

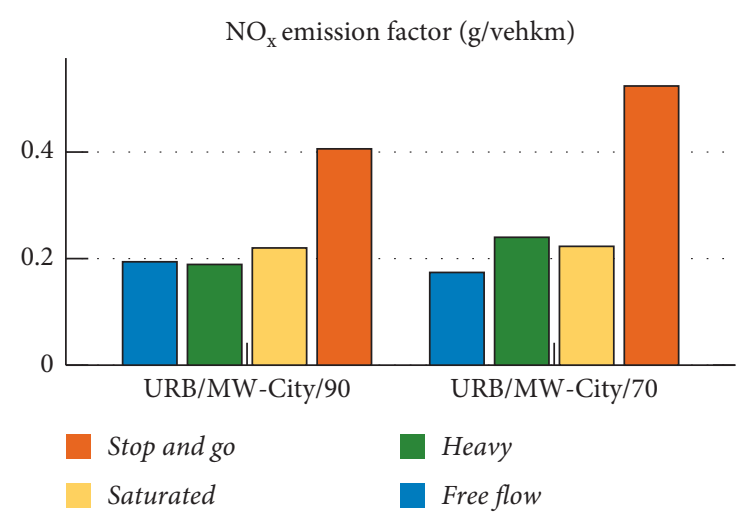

(b)

Figure 1: HBEFA emission factors for $\mathrm{CO}$ and $\mathrm{NO}_{\mathrm{x}}$ [19].

$$
Q_{a}=\sum_{r \in \mathscr{R}} \sum_{s \in \mathcal{S}} \sum_{k \in \mathscr{K}^{r s}} f_{k}^{r s} \delta_{a, k}^{r s}
$$

where $f_{k}^{r s}$ is the number of travellers that chose the path $k$ between origin $r$ and destination $s$ and $\delta_{a, k}^{r s}$ is the so-called incidence matrix, having the value of 1 if link $a$ is part of path $k$ between $r$ and $s$ and 0 otherwise. Solving problem $((1 \mathrm{a}))-(1 \mathrm{~d}))$ will lead to optimal path demand $f_{k}^{* r s}$ and link flows, $Q_{a}^{*}$, where the travel cost between each O-D pair, $r s$, is the minimum possible and equal for every $k \in \mathscr{K}^{r s}$ with positive flow.

The link travel time $t_{a}$ is considered as a function of link volume $Q_{a}$, known as the link cost function or Volume Delay Function (VDF). VDF provides a mathematical relationship between mean unit travel time, $t_{a}$, and traffic volume, $Q_{a}$, representing the measure of the disutility of each corresponding link. Beckmann also proved that the problem ((1a)-(1d)) has a unique solution if and only if

$$
\begin{aligned}
& \frac{\partial t_{a}\left(Q_{a}\right)}{\partial Q_{b}}=0, \quad \forall a \neq b, \\
& \frac{\partial t_{a}\left(Q_{a}\right)}{\partial Q_{a}}>0, \quad \forall a .
\end{aligned}
$$

Therefore, according to equation (2), the travel time on each link should not depend on the flow on other links, and according to equation (3), VDF should be strictly convex. The latter means that travel time should only increase while volume increases. Specifically, in most of the cases, travel time strictly increases with the volume capacity ratio $(V / C)$, $Q_{a} / C_{a}$. The function named Bureau of Public Roads (BPR, equation (4)) is a commonly used function of this type:

$$
t_{a}\left(x_{a}\right)=t_{0}\left(1+\gamma\left(\frac{Q_{a}}{C_{a}}\right)^{\theta}\right),
$$

where $t_{0}$ is free flow travel time and $\gamma$ and $\theta$ are parameters.

HBEFA emission factors are given as a discrete functions of speed and/or traffic flow. When an environmental analysis is based on STA outputs, traffic volume is usually not used directly, but as a ratio of volume to capacity $V / C$. In this study, we use $V / C$ thresholds (Table 1) presented in Trafikverket [27].

The assigned flow obtained from the solution of problem $((1 \mathrm{a})-(1 \mathrm{~d}))$ is used to determine the emission factor, $e_{a}^{p}\left(Q_{a}^{*} / C_{a}\right)$, for every link $a$ and pollutant $p$ in $\mathrm{g} / \mathrm{vehkm}$. Next, the emission factor multiplied by the traffic activity expressed in vehicle kilometre travelled, $Q_{a}^{*} \cdot l_{a}$, gives the total grams of each pollutant, $p$, emitted at link $a, E_{a}^{p}$ :

$$
E_{a}^{p}=e_{a}^{p}\left(\frac{Q_{a}^{*}}{C_{a}}\right) \cdot Q_{a}^{*} \cdot l_{a} .
$$

2.3. Location-Specific Effects of Emissions. A volume delay function should satisfy the conditions expressed by equation (2). Any link interactions and thus traffic flow phenomena, such as spill-back, are therefore neglected. The latter can result in an inaccurate modelling of congestion's level and location. However, several studies [28-30] have highlighted and demonstrated that increased traffic congestion levels leads to high emission rates. Besides the congestion's level, its location can also be an influential factor as emissions have location-specific effects.

The estimated emissions derived from the traffic analysis together with the so-called dispersion models determine the air quality at a specific location. The accurate estimation of location and extent of congestion then becomes even more important, considering the nature of the dispersion. Based on the mass of pollutant emitted per vehicle, mathematical and physical models describe the dispersion of the pollutant, leading to pollutant concentrations at specific locations. One of the simplest and most frequently used approaches of dispersion modelling is the street canyon model [31], where the pollutants' concentration depends on the so-called aspect ratio, which is the height of road side buildings by the width of the road. Meteorological conditions, such as the wind speed, are also an important factor affecting the dispersion of the vehicular emissions. In addition, different network links are associated with different number of pedestrian and cyclists affected by the emissions. Together with the pedestrians and cyclists' exposure, the exposure of people living or working by the roadside is also a local 
TABLE 1: Volume/capacity ratio thresholds for the traffic situation determination [27].

\begin{tabular}{llccc}
\hline $\begin{array}{l}\text { Speed limit } \\
(\mathrm{km} / \mathrm{h})\end{array}$ & $\begin{array}{l}\text { Free } \\
\text { flow }\end{array}$ & Heavy & Saturated & Stop and go \\
\hline 90 & $<0.65$ & $0.65-0.85$ & $0.85-1.35$ & $\geq 1.35$ \\
70 & $<0.39$ & $0.39-0.84$ & $0.84-1.35$ & $\geq 1.35$ \\
$<50$ & $<0.52$ & $0.52-0.78$ & $0.78-1.22$ & $\geq 1.22$ \\
\hline
\end{tabular}

Each row is associated with a different road type indicated by the speed limit.

characteristic. Usually, at a street canyon, the high buildings are associated with denser population, and therefore alongside the increased pollutants' concentration, more people are affected [32]. This could have a direct effect during an economic analysis, such as a cost-benefit analysis, where the monetary cost of a gram of pollutant emitted is analogous to the number of people exposed and affected [9].

Therefore, applications of emission modelling such as dispersion and exposure modelling may be sensitive to the spatial allocation of the emission rates. A necessity of spatially accurate emission estimation is thus emerged, contradicting the STA assumptions about the location of congestion. It should be noted here that not all the emitted pollutants have local effects, for instance, $\mathrm{CO}_{2}$ has long-term and global effects, and therefore the spatial distribution of emissions is not of high importance in this case.

2.4. Postprocessing of Static Model Outputs. As static models cannot describe dynamic phenomena of traffic flow, there is a risk of introducing considerable errors, especially in conjunction with air quality and exposure modelling. Conversely, dynamic traffic models include components to model dynamic phenomena at various levels and might therefore be better suited for estimating emissions. However, such models cannot guarantee a unique equilibrium solution and often are unsuitable for policy analyses. Also, they are more time consuming to calibrate and run and require a high amount of input data. An alternative approach with lower computing cost is to postprocess the static models' outputs in order to provide a more detailed representation of traffic conditions and thus more accurate emissions.

The problem of using outputs from static models in emission estimation has been addressed in the literature by various postprocessing techniques. One approach is congestion correction techniques, capable to reflect the spatiotemporal variability of speeds [11,33-35]. The approach suggested by Negrenti [33] is based on a congestion correction factor, representing the speed variability along a link, which can improve the accuracy of emissions estimations. Additionally, the proposed model in Nesamani et al. [34] can provide a more precise prediction of emissions by capturing traffic variations. Focusing on a set of factors that correlate congestion on each link, a dynamic variation in speed was constructed. Smit et al. [11] also highlighted the significant underestimations of emissions when using assignment outputs such as the average link speed. They identified that speed may vary on a link, supporting that the emission estimation can be improved by using speed distributions instead of a mean link speed. Finally, Ryu et al. [35] suggested a corrected average speed model by analysing the traffic characteristics that can cause serious emission estimation errors.

Bai et al. [4] developed direct speed postprocessing techniques for improving emission estimates derived from the STA, based on intersection delays and queuing analysis approaches. Their methods mainly rely on the alternative forms of speed-flow curve and queuing analysis proposed in Akcelik [36] and Dowling and Skabardonis [37]. They concluded that different postprocessors generate dissimilar impacts on emissions. However, spillback phenomena are not taken into account by these methods, as queues are represented as vertical points, which is noted by Dowling and Skabardonis [37].

A common characteristic of the presented postprocessing techniques is that they focus solely on single link speeds, without considering network topology and link interactions. Thus, queue spillback is not taken into account which may also lead to an erroneous location of congestion. An alternative approach would be to consider a postprocessing technique which includes a spatial description of the queuing in the network. This could be achieved by applying a one-shot simulation-based network loading, relying on the known route choices from the static model. Micro, meso, or macro dynamic traffic simulation approaches could be used for this purpose, but with the downside of increased computational time and dynamic demand as input. Depending on the loading technique, various levels of additional calibration would be required. Even the macroscopic approaches would require time-dynamic input in order to be able to reproduce congestion patterns. Nevertheless, quasidynamic network loading approaches allow for link interaction to be taken into account, without employing a full-time dynamic model. Such approaches have been applied with static models in Bliemer et al. [16] and Bundschuh et al. [17] for improving travel time estimation on routes. The output from such approaches are, however, not straightforward to apply to macroscopic emission models.

\section{Analysis of Errors Originating from Static Traffic Models}

The purpose of this section is to identify and quantify errors introduced by a static model into the emission estimation procedure. We distinguish these errors by their source into two major categories: errors due to aggregated traffic conditions both at a temporal and spatial level (Section 3.2) and errors coming from the static loading and the incorrect location of congestion (Section 3.3). Section 3.1 describes our methodology by introducing the case study.

3.1. Methodology. A 19-km long section of the E4 motorway, located north of Stockholm city, has been adopted for our case study (see Figure 2). We divided the section in 27 homogeneous links based on the location of the on- 


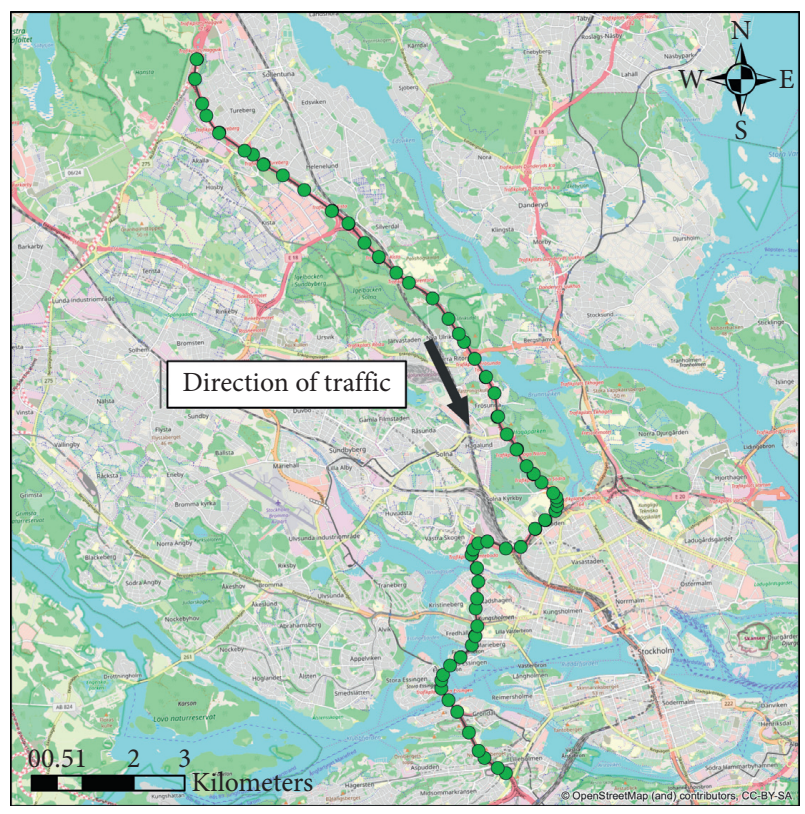

- Motorway section

- Radar sensors

Figure 2: Modelled motorway section and the sensors' position.

ramps and off-ramps. During the morning peak, one of the links becomes an active bottleneck resulting in spillback of queues over several upstream links. For this reason, we set our analysis period to be the morning peak between 6:30 and 9:30, which is a long enough time interval for the complete formation and dissolution of queues. Additionally, this motorway section is densely equipped with radar sensors, providing one-minute average speed and flow per lane. The sensor data provide a sufficiently realistic description of the traffic conditions, and thus we used these data to construct the ground truth for our experiments. Specifically, we consider counts from three months (January, February, and March of 2013). We initially estimate our ground-truth emissions for the motorway section based on the traffic state and traffic activity obtained by the radar sensors' counts of speed and flow. Next, for the same stretch, we estimate emissions based on $V / C$ ratio thresholds resulting from a static model, considering a stable demand during the analysis period. By comparing these emissions with the ground truth, we can estimate the errors that static traffic models introduce in the emission modelling.

To construct a more reliable ground truth together with sensors data we use a traffic state estimator. Radar sensors are stationary, providing cross-sectional data, available only at specific locations (the sensors are located approximately at every 500 meters). In addition, the measurements are noisy, and since emissions are not linearly related to speed, a small error in the speed counts can cause considerable errors regarding the emissions. Therefore, we apply a traffic state estimator to reconstruct the traffic state, reduce the measurements noise, and accordingly obtain a more reliable ground truth for our experiments.
The traffic estimator is based on the spatiotemporal interpolation and smoothing approach suggested and described by Treiber and Helbing [38] and Treiber and Kesting [39]. The method relies on a discrete convolution, with a symmetric exponential function as the weighted kernel, operating actually as a low-pass filter, smoothing temporal variation, and spatial fluctuations. More specifically, we used the adaptive smooth method (ASM) $[38,39]$, where two smoothed fields of the traffic variables are considered, concerning different propagation velocities, one for free and one for congested traffic. The estimator takes into account that information in free-flowing conditions travels downstream, while in congested conditions perturbations propagate upstream. The resulting average traffic variable value is a superposition of the two fields. The final output of the interpolation method is a complete speed, $V_{\kappa, \tau}$, and flow, $Q_{\kappa, \tau}$, field as a function of the discretised space $\kappa$ and time $\tau$. Let the lengths of the space and time discretisation intervals be denoted by $\lambda$ and $\mu$, respectively. Figure 3 illustrates the reconstructed speed field, obtained from the ASM traffic estimation model with $\lambda=1$ meter and $\mu=1$ minute, for the morning peak hours of a typical day at the motorway stretch under investigation. The $y$-axis denotes the location of each discretised space interval, with the reference point 0 being the starting point of the segment, at the most north point in Figure 2. Accordingly, $x$-axis denotes the discretised time during the morning peak hours.

In practice, there is a need to use longer discretisation intervals, due to both computational burden and emission modelling issues. Regarding the latter, HBEFA emission factors are not instantaneous, and they rather represent aggregated situations relying on average conditions during a driving cycle. Therefore, they should be applied on stable traffic conditions. We chose the spatiotemporal interval lengths of $\lambda=50$ meters and $\mu=5$ minutes, assuming that they are representative of the HBEFA traffic situations and at the same time the traffic variations can be sufficiently characterised.

The V/C thresholds of the HBEFA traffic situations are developed to be used with static model outputs. The threshold for the stop and go situation (see Table 1) can be greater than 1 . This is because STA models allow the volume to be higher than the capacity of the road; the underlying VDF represents travel times as a function of demand rather than the actual flow. Thus, the $V / C$ thresholds as illustrated in Figure 4(a) cannot directly be applied to the ASM output.

In order to apply the definition of HBEFA emission factors, we suggest to distinguish the different traffic situations based on the fundamental diagram. The fundamental diagram more realistically captures the relationship between flow, density, and speed. In contrast to VDF, the relationship between flow and speed is not monotonic. Low flows can correspond to either low speeds, in the case of saturated conditions, or to high speeds, in the case of nonsaturated conditions. Thus, flow by itself is not sufficient to determine the traffic situation and we cannot apply the $V / C$ depended emission factors, $e^{p}$. For this reason, we translate the $V / C$ ratio thresholds into speed thresholds, using a hyperboliclinear fundamental diagram suggested by Work et al. [40]. 


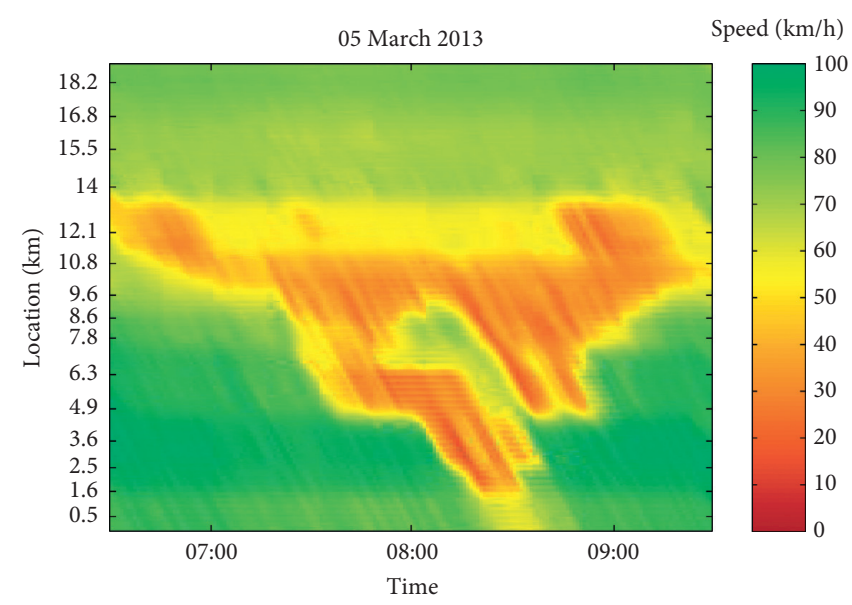

FIGURE 3: Speed field as a function of space and time, for the morning peak hours of a typical day.

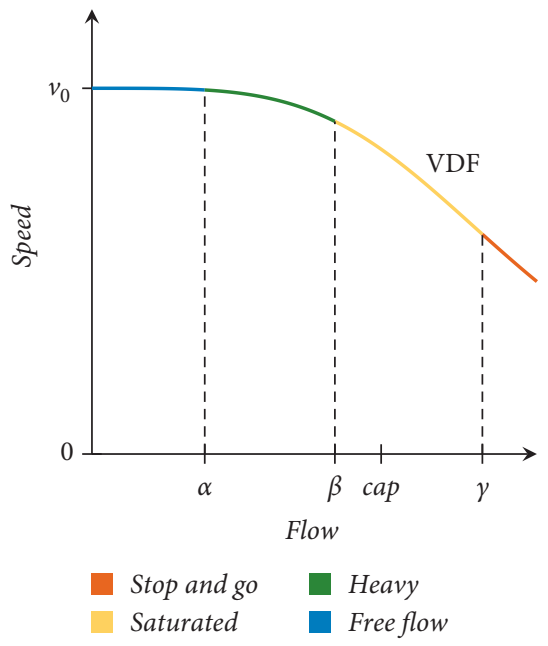

(a)

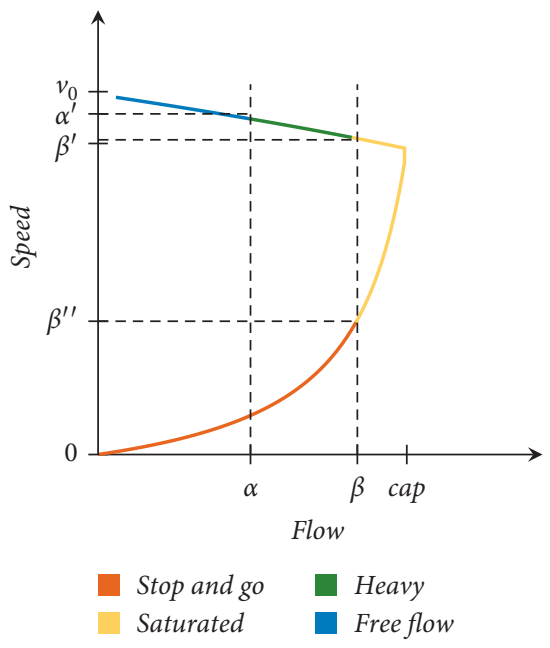

(b)

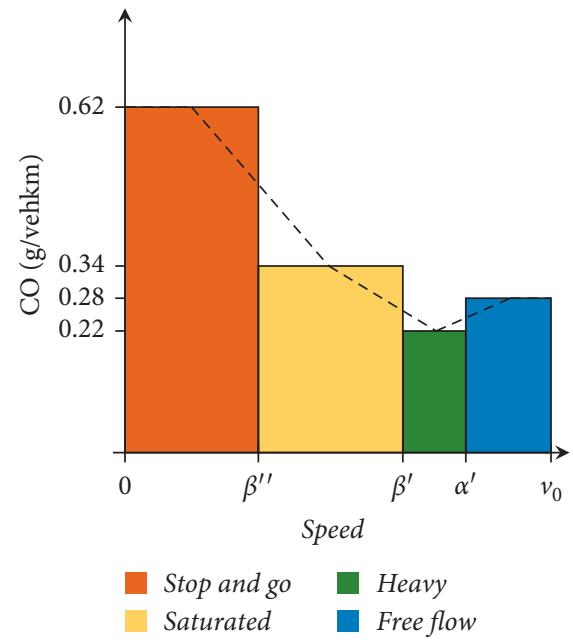

(c)

FIgURE 4: (a) Volume to capacity thresholds and VDF (flow $<\alpha$ : Free flow, $\alpha \leq$ flow $<\beta$ : Heavy, $\beta \leq$ flow $<\gamma$ : Saturated, and flow $\geq \gamma$ : Stop and go). (b) Hyperbolic linear fundamental diagram and derivation of speed thresholds [19] (speed $>\alpha^{\prime}:$ Free flow, $\alpha^{\prime} \geq$ speed $>\beta^{\prime}:$ Heavy, $\beta^{\prime} \geq$ speed $>\beta^{\prime \prime}$ : Saturated, and speed $\leq \beta^{\prime \prime}$ : Stop and go). (c) CO emission factor as a discrete (bars) and as a continuous (dashed line) function of average speed.

Therefore, emission factors are now expressed as a function of average speed, $g^{p}$. Figure 4(b) illustrates this process, where the flow thresholds are projected to the speed axis to determine the speed thresholds. A more detailed description of the process of deriving speed thresholds can be found in Tsanakas et al. [19].

Based on the reconstructed speed and flow space-time field, we are now able to compute the corresponding emission space-time field for each pollutant. Let $V_{\kappa, \tau}^{\eta}$ and $Q_{\kappa, \tau}^{\eta}$ be the estimated from ASM average cell speed and flow in $\mathrm{km} / \mathrm{h}$ and veh/h respectively, where $\eta$ is the day index, $\eta \in \mathscr{H}$, and $\mathscr{H}$ a set of the considered days. As ground truth, we assume the average emissions among 35 representative weekdays belong into the three months period (January, February, and March 2013). The daily average mass in grams of pollutant, $p$, emitted at each space and time interval, $\kappa, \tau$, can be estimated as

$$
E_{\kappa, \tau}^{p}=\frac{1}{|\mathscr{H}|} \sum_{\eta \in \mathscr{H}}\left[Q_{\kappa, \tau}^{\eta} \cdot \lambda \cdot \mu \cdot g_{\kappa}^{p}\left(V_{\kappa, \tau}^{\eta}\right)\right]
$$

where $\lambda$ and $\mu$ are the length of the discretisation space and time intervals in $\mathrm{km}$ and $\mathrm{h}\left(\lambda=50 / 10^{3} \mathrm{~km}, \mu=5 / 60 \mathrm{~h}\right)$ and $g_{\kappa}^{p}\left(V_{\kappa, \tau}\right)$ is the emission factor of pollutant $p$ in $\mathrm{g} / \mathrm{vehkm}$, as function of average speed $V_{\kappa, \tau}$ according to the speed limit of $\kappa$ space interval. A more detailed description of emission estimation based on ASM reconstructed fields can be found in Tsanakas et al. [41].

3.2. Errors of Emission Estimations due to Spatiotemporal Aggregation. As we have mentioned in the introduction of this section, we categorise the errors that static modelling can introduce to the emission estimation in two types: errors that concern the spatiotemporal resolution of average speed 
and flow and errors related to inaccurate congestion location. If we assume that STA can accurately predict the traffic conditions, similar to the ASM, but aggregated over longer spatial and temporal intervals, we can isolate the errors that concern the first category. We hypothesise that longer aggregation intervals influence the emission estimations as emission factors have a nonlinear relationship with the average speed. To test our hypothesis, we first aggregate the estimated by ASM flows, $Q_{\kappa, \tau}^{\eta}$, and speeds, $V_{\kappa, \tau}^{\eta}$, spatially and temporally, over a link level and a three-hour level, respectively. Let $\bar{Q}_{\kappa, \tau}^{\eta}$ and $\bar{V}_{\kappa, \tau}^{\eta}$ be the aggregated flows and speeds, respectively, given by

$$
\begin{aligned}
& \bar{Q}_{\kappa, \tau}^{\eta}=\frac{1}{\left|\mathscr{D}_{a, \kappa}\right||\mathscr{T}|} \sum_{\kappa^{\prime} \in \mathscr{D}_{a, \kappa}} \sum_{\tau^{\prime} \in \mathscr{T}} Q_{\kappa^{\prime}, \tau^{\prime}}^{\eta}, \\
& \bar{V}_{\kappa, \tau}^{\eta}=\frac{1}{\left|\mathscr{D}_{a, \kappa}\right||\mathscr{T}|} \sum_{\kappa^{\prime} \in \mathscr{D}_{a, \kappa}} \sum_{\tau^{\prime} \in \mathscr{T}} V_{\kappa^{\prime}, \tau^{\prime}}^{\eta},
\end{aligned}
$$

where $\mathscr{T}=\left\{1,2, \ldots, n_{\tau}\right\}$ and $n_{\tau}$ is the number of the time intervals contained in the analysis period $\left(n_{\tau}=36\right.$ in this case), $a$ is the link that includes $\kappa$, and $\mathscr{D}_{a, \kappa}$ is the set of the space intervals included in link $a$. Then, based on such averaged traffic conditions, we estimate the emissions similar to equation (6) as

$$
\bar{E}_{\kappa, \tau}^{p}=\frac{1}{|\mathscr{H}|} \sum_{\eta \in \mathscr{H}}\left[\bar{Q}_{\kappa, \tau}^{\eta} \cdot \lambda \cdot \mu \cdot g_{\kappa}^{p}\left(\bar{V}_{\kappa, \tau}^{\eta}\right)\right] .
$$

If we assume that an STA model is accurate and the errors are only related to the spatiotemporal aggregation, the emissions $\bar{E}_{\kappa, \tau}^{p}$ correspond to the emissions derived from this "perfect" STA model. To quantify the influence of using longer aggregation intervals, we compare the emissions $\bar{E}_{\kappa, \tau}^{p}$ with the ground truth, $E_{\kappa, \tau}^{p}$. For a more illustrative way to present our result, we introduce here the term Emission Errors due to Spatial and Temporal Aggregation, ESTA, given by

$$
\operatorname{ESTA}_{\kappa, \tau}^{p}=\frac{\bar{E}_{\kappa, \tau}^{p}-E_{\kappa, \tau}^{p}}{E_{\kappa, \tau}^{p}} 100
$$

ESTA represent the percentage emission over or underestimations, caused by spatiotemporal aggregation, for pollutant, $p$, and for each space and time interval, $\kappa, \tau$.

Being consistent with our initial hypothesis, Figure 5(a) demonstrates that longer aggregation periods significantly affect the emission estimation. The underestimation at specific locations can reach up to $40 \%$ for pollutants such as $\mathrm{HC}$ and $\mathrm{CO}$ which are more sensitive to the variations of traffic conditions. The higher underestimations are mainly located where the congestion is observed, namely, upstream the bottleneck. Speed variations are higher at the congested parts, and thus there is a clear correlation between ESTA and congestion. Moreover, the error depends on the link length, since in long links the spatial average speed may not be representative of the link's conditions. Therefore, even if we assume that a static model would accurately predict the same speeds and flows with the measurements, there are still substantial errors because of the low spatiotemporal resolution.

One possible source of such errors is the discrete nature of the HBEFA emission factors. Slight changes in traffic state may trigger a transition to another traffic situation and generate significant differences in terms of emissions. In order to avoid large changes among the emissions factors for small changes in the traffic state, we suggest a continuous version of HBEFA. We derive the new continuous emission factors, $\bar{e}^{p}(Q / C)$ and $\bar{g}^{p}(V)$, relying on the linear interpolation between emission factors that correspond to adjacent traffic situations, similar to the interpolation performed by Wismans et al. [15]. By applying the continuous version of HBEFA, the errors caused by the temporal resolution can be significantly reduced. According to Figure 5(b), the emission calculations become less sensitive to the aggregation period when the interpolated version is used. We should highlight here that the subsequent analyses described in the next sections are performed based on the continuous emission factors.

Figure 6 illustrates the space-time emission field, when the HBEFA emission factors are given as continuous function of speed. Considering this figure, it would be helpful here to give a small description of the network's traffic situation during the morning peak. The upstream end of the bottleneck link is located at the position of $10.8 \mathrm{~km}$, where it is also the starting point of the downstream propagated queues. The four lanes upstream this point, become two downstream, decreasing the capacity and creating a lane-drop bottleneck. The bottleneck is mainly active during the second hour of our analysis period. The position of $12.1 \mathrm{~km}$ is associated with the downstream end of the bottleneck link.

3.3. Errors of Emission Estimations due to Static Loading. In this section, we attempt to identify and quantify emission errors which come from the unrealistic location of congestion modelled by static models. For this reason, we compare our ground truth with emission estimations derived from a static loading procedure for the same case study segment. It should be highlighted here that we focus only on the loading phase of an STA model; there are no route choice alternatives in the considered segment. In this way, we can isolate the errors caused by the static loading and exclude any possible error coming from an inaccurate route-choice modelling.

Given that there is no queue formed before the start of the first hour as well as that all the queues have been discharged after the end of the third hour of the analysis period, we assume that the three-hour demand is equal to the total three hours inflow of the segment. Hence, the three-hour OD matrix is obtained by the observed inflow from the radar sensors located at the starting point of the segment and at each on-ramp. However, according to Figure 6, in reality, the bottleneck is active only during the second, intermediate hour of the considered analysis period. Traffic conditions during the first and the third hour are stable, and since no dynamic traffic flow phenomena were observed, we assume 


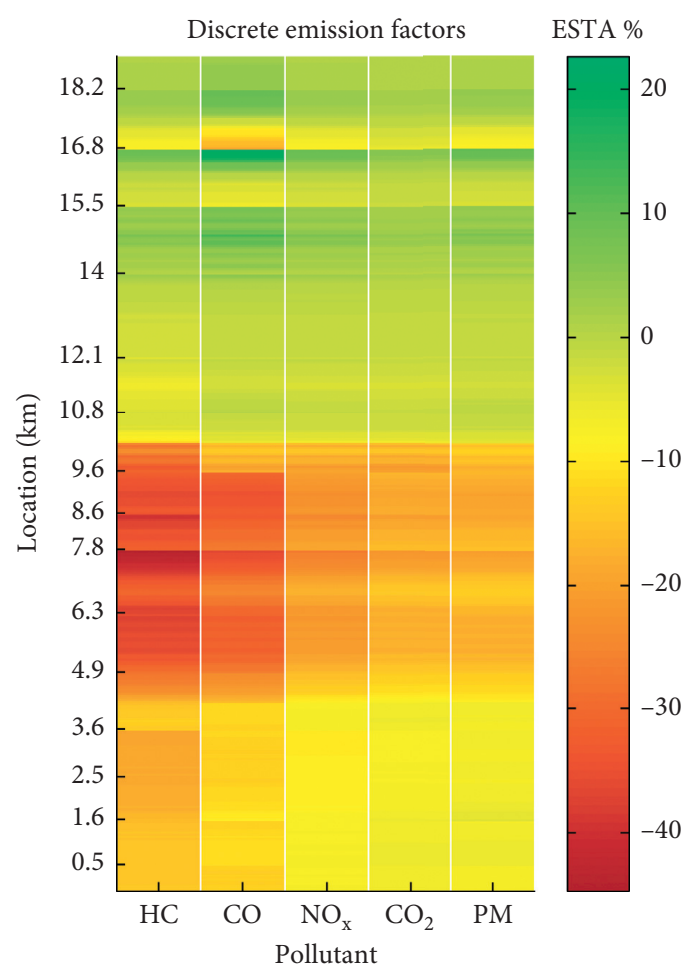

(a)

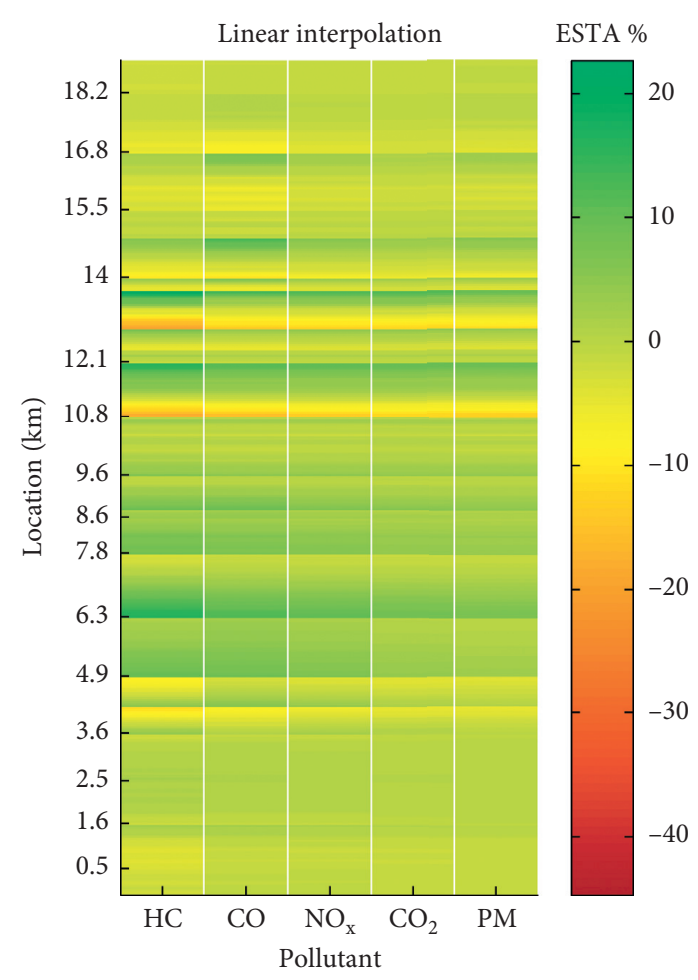

(b)

FIgURE 5: Average emission errors due to spatial and temporal aggregation (ESTA) when HBEFA emission factors are expressed as (a) discrete and (b) continuous functions of average speed.

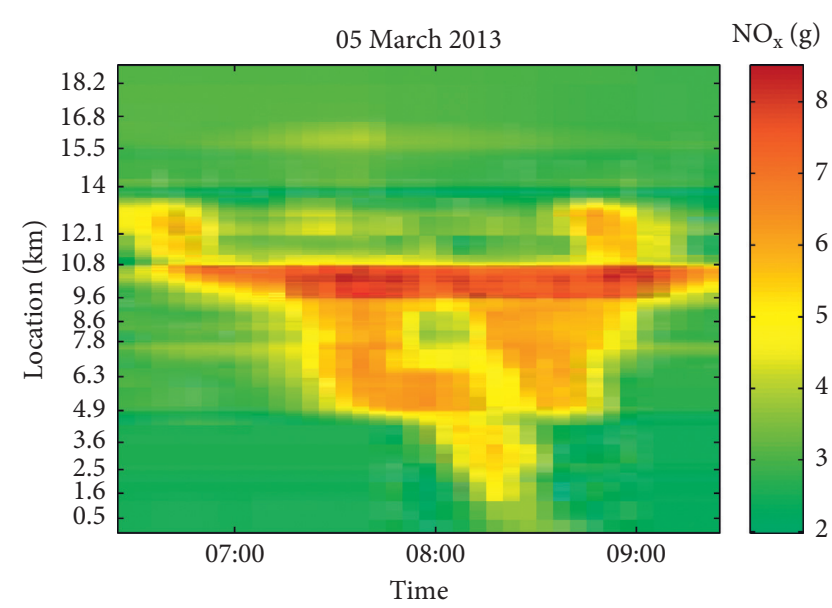

Figure 6: $\mathrm{NO}_{\mathrm{x}}$ space-time field using the linearly interpolated version of HBEFA, for the morning peak hours of a typical day.

that a static model can lead to sufficiently accurate emission estimations. Therefore, the first and the third hour are excluded from our experiments. The OD-matrix period is split, then, into three smaller, one-hour OD matrices based on the demand variation indices suggested by Björketun and Carlsson [42]. Specifically, to the first hour (6.30-7.30), 27\% of the total demand was assigned, while to the second (7.30-8.30) and the third (8.30-9.30), 37.5\% and $35.5 \%$ of the total demand were assigned, respectively. The capacities, as well as the other parameters of the static model, are estimated from calibration using the flow and the speed counts from the radar sensors.

For a comprehensible illustration of the results, we introduce the term Excess due to Congestion Emission factors $\mathrm{ECE}_{\kappa}^{p}$, defined for the ground truth as

$$
\mathrm{ECE}_{\kappa}^{p}=\frac{1}{|\mathscr{H}||\mathscr{T}|} \sum_{\eta \in \mathscr{H}} \sum_{\tau \in \mathscr{T}} \bar{g}_{\kappa}^{p}\left(V_{\kappa, \tau}^{\eta}\right)-\bar{g}_{\kappa}^{p}\left(V_{\kappa}^{0}\right),
$$

while the percentage ECE is given by

$$
\operatorname{ECEP}_{\kappa}^{p}=\left(\frac{1}{|\mathscr{H}||\mathscr{T}|} \sum_{\eta \in \mathscr{H}} \sum_{\tau \in \mathscr{T}} \bar{g}_{\kappa}^{p}\left(V_{\kappa, \tau}^{\eta}\right)-\bar{g}_{\kappa}^{p}\left(V_{\kappa}^{0}\right)\right) \frac{100}{\bar{g}_{\kappa}^{p}\left(V_{\kappa}^{0}\right)},
$$

where $V_{\kappa}^{0}$ is the free flow speed of space interval $\kappa$ and $\bar{g}_{\kappa}^{p}\left(V_{\kappa}^{0}\right)$ is the emission factor, if at time $\tau$ and space $\kappa$ there was not any congestion. Therefore, ECEP expresses the differences over space in emission estimates between the current situation and the hypothetical situation where there would be no congestion at all. In this way, we can isolate the additional emissions exclusively originated from congestion and obtain their exact location. We select to express our results here in terms of ECEP in order to show the effect that the location of congestion can have to the emission estimates. Accordingly, in the case of an STA model, ECEP can be computed as

$$
\operatorname{ECEP}_{a}^{p}=\frac{\bar{e}_{\kappa}^{p}\left(Q_{a}^{*} / C_{a}\right)-\bar{e}_{\kappa}^{p}(0)}{\bar{e}_{\kappa}^{p}(0)} 100
$$


The optimal link volumes, $Q_{a}^{*}$, regard averaged in time and space (over a link-level) traffic conditions. Thus, errors due to spatiotemporal aggregation may also be reflected in STA ECEP. In order to mitigate these errors and isolate the effect of the loading procedure, we employ here the continuous emission factors, both for the ground truth in equation (11) and STA ECEP in equation (12). As we show in the previous section, using such continuous emission factors can effectively moderate the errors due to spatiotemporal aggregation.

Figure 7 illustrates the value of ECEP at every location of the segment for each one of the pollutants. Considering the ground truth (Figure $7(\mathrm{a})$ ), the excess emissions due to congestion are located between the positions of $10.8 \mathrm{~km}$ and $9.6 \mathrm{~km}$, namely, at the first links upstream the bottleneck. Additionally, there are also some excess emissions upstream the location of $9.6 \mathrm{~km}$ because of the queue spillback. Especially, for some pollutants sensitive to congestion, such as $\mathrm{HC}$, the excess emissions are high even for the first kilometres of the segment.

In contrast to the ground truth, the static model, without considering any capacity or density constraints, assigns all the delays at the bottleneck link, due to the link's high $V / C$ ratio (equation (4)). Consequently, the excess emissions are also gathered at the same location, at the bottleneck link. Figure 7(b) demonstrates this fact since the highest excess emissions are located between the positions of $10.8 \mathrm{~km}$ and $12.1 \mathrm{~km}$, namely, at the bottleneck link. Static loading is not able to capture the propagation of the queues, neglecting an important part of the network's emissions.

It also becomes interesting that according to the static model, higher than the ground-truth excess emissions, especially for $\mathrm{CO}$, are estimated downstream the bottleneck. The demand ratio may be high compared to the capacity for some links downstream the bottleneck, but in reality, the inflow rate at those links is restricted to the bottleneck's capacity. The static model, by not taking any capacity constraint into account, loads the entire demand instantaneously and neglects the metering effect, overestimating the emissions downstream the bottleneck.

Although the differences in the total network's emissions, between the ground truth and static loading, are low (the differences range from $1.30 \%$ to $1.42 \%$ depending on the pollutant), the location of excess emissions differs significantly, leading to the effects discussed in Section 2.3. Therefore, from an emission modelling perspective, an ideal model should be able to propagate the queues and as well as to block the paths after the bottleneck up to bottlenecks capacity, leading to a more realistic location of congestion.

\section{Quasidynamic Loading as a Postprocessing Technique}

In the previous section, we show that the use of static traffic models in emission modelling emission may lead to considerable errors, related mainly to the location of congestion. For a more reliable modelling of emissions of spatial distribution, we suggest the use of a quasidynamic loading model as a postprocessor over the static assignment results.
In this way, the route choice is based on the traditional VDF considered by STA, providing the required link inflows for the network loading, which is not static anymore but quasidynamic. The distribution of the static demand, $q_{r s}$, along the paths $k, k \in \mathscr{K}^{r s}$, can therefore be computed solving problem $((1 \mathrm{a})-(1 \mathrm{~d}))$ similar to STA. Assume that $\mathscr{D}^{r s, k}$ is the set containing the consecutive links of path $k$, $k \in \mathscr{K}^{r s}$. The term quasidynamic means that part of the optimal path demand $f_{k}^{* r s}$ is loaded in the path $k$ instantaneously, similar to the static case, until a link flow, $Q_{a}$, $a \in \mathscr{D}^{r s, k}$, reaches its capacity, $C_{a}$. Then, path $k$ is blocked at the upstream end of the link and the rest of the demand $f_{k}^{*}$ ss is forming a queue propagating upstream of link $a$ dynamically. Real-time variable does not exist, but the propagation of the queues is based on pseudo time from the time that the flow of link $a$ reaches the capacity $C_{a}$. Our hypothesis now is that excess due to congestion emissions can be estimated more accurately by modelling propagation of queues during the loading phase.

4.1. Static Assignment with Queuing. Static assignment with queuing (STAQ) [16] is a quasidynamic model which employs traffic flow dynamics, but at the same time, it considers two important static assumptions. The network loading submodel consists of two phases, the squeezing and the queuing phase. During the squeezing phase, each path share $f_{k}^{* r s}$ of the static (first static assumption) demand, $q_{r s}$, is loaded instantaneously (second static assumption) into the network through a path-based network loading model [43], which ensures that no link flow, $Q_{a}, a \in \mathscr{D}^{r s, k}$, exceeds capacity. The squeezing phase is formulated as a fixed point problem which is iteratively solved $[43,44]$, aiming at determining a set of consistent, path-desegregated sending and receiving flows for any network node.

The queuing phase is based on the LWR first-order traffic flow model $[45,46]$. Traffic flow is treated as a one-dimensional compressible fluid, and the basic dynamic variables, in correspondence to fluid dynamics, are the density $\rho(x, t)$, the flow $Q(x, t)$, and the mean speed $V(x, t)$, with the independent variables being the location $x$ and the time instant $t$. The conservation law defines the basic relationship between flow and density as

$$
\frac{\partial \rho}{\partial t}+\frac{\partial Q}{\partial x}=0
$$

describing how a change in density over time relates to a change in flow over space. Lighthill and Whitham [45] and Richards [46] introduced a static relation between flow and density, $Q=Q_{f}(\rho)$, known as the fundamental diagram of traffic flow. Therefore, the conservation law becomes

$$
\frac{\partial \rho}{\partial t}+\frac{\mathrm{d} Q_{f}(\rho)}{\mathrm{d} \rho} \frac{\partial \rho}{\partial x}=0 .
$$

The space-mean speed, $V$, is computed by the hydrodynamic flow relation, $V=Q / \rho$, considering the assumption that traffic conditions at location $x$ and time $t$ are in steady state. Solving equation (14) with the method of characteristics curves $[47,48]$ implies that traffic state remains 


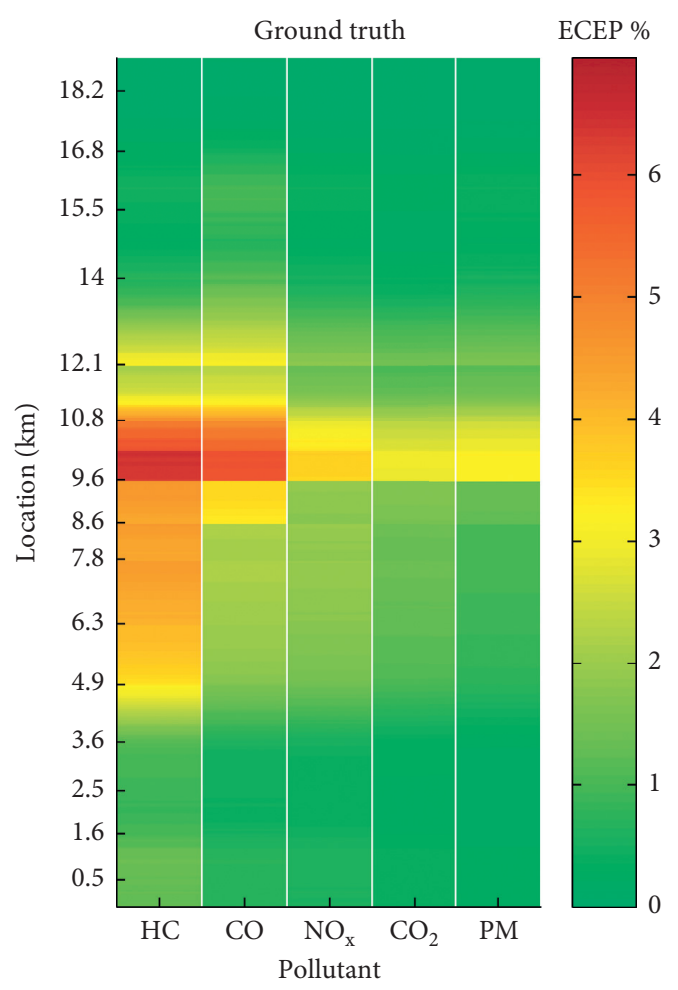

(a)

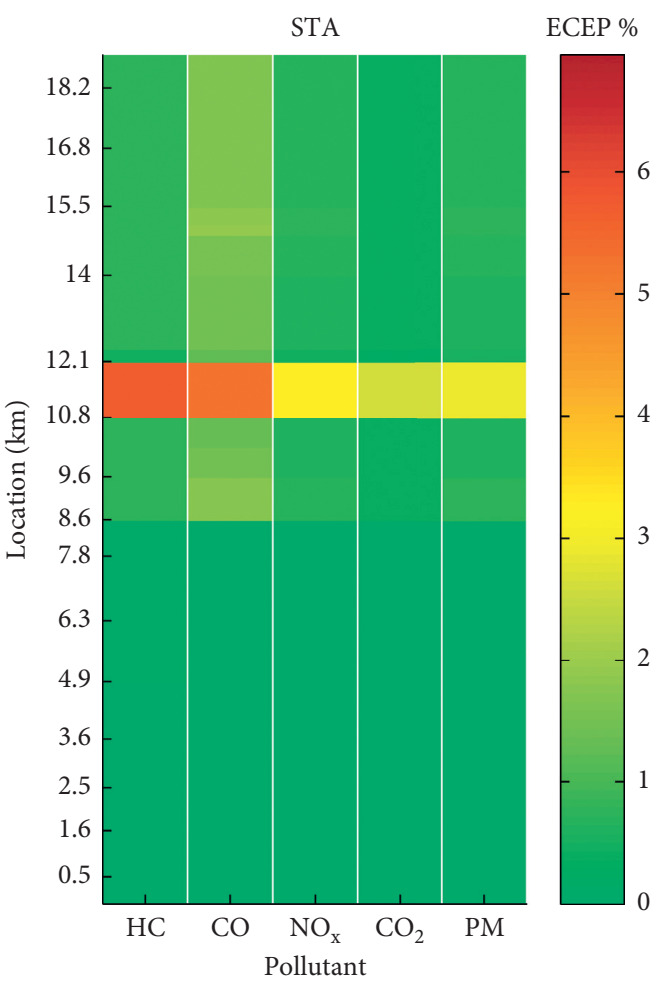

(b)

Figure 7: Excess due to congestion emissions based on (a) the ground truth and (b) static loading.

constant along a characteristic curve or wave. At the boundary between two different traffic states, a shock wave is formed propagating with a velocity of

$$
w=\frac{\mathrm{d} Q_{f}(\rho)}{\mathrm{d} \rho} .
$$

Daganzo [49] proposed the Cell Transmission Model (CTM) which constitutes a time and space discrete numerical solution scheme for the LWR model, where space is divided in homogeneous cells. Accordingly, Link Transmission Model (LTM) [50] is a discrete time model, but instead of cells, space is now divided into links. Yperman adopted the simplified wave theory suggested by Newell [48], an alternative way to keep track of the traffic state by relying only on link boundary conditions.

Figure 8 illustrates the way that the link model of LTM propagates the traffic states in a network using equation (14) and the simplified wave theory of Newell [48]. Consider two simple consecutive links $j$ and $j+1$, where the inflow of link $j$, for the state $1, Q_{j}^{1}$, is greater than the capacity of link $j+1$, $C_{j+1}$. Because link $j+1$ cannot receive flow greater than its capacity, the actual flow that will be transmitted from link $j$ to link $j+1, Q_{j}^{2}$, equals to the capacity of link $j+1, C_{j+1}$. LTM blocks the link's upstream boundary up to link's capacity, by applying a node model that is associated with node capacity, sending and receiving flows, similar to the supplydemand method used in CTM. Therefore, $C^{j+1} \Delta t$ vehicles are actually transmitted during a time period $\Delta t$ from link $j$ to link $j+1$ (sending flow), and the remaining vehicles,
$\left(Q_{\alpha}^{1}-C_{j+1}\right) \Delta t$, form a queue (state 2$)$, with higher density $\rho_{j}^{2}>\rho_{j}^{1}$. The fundamental diagram of Figure 8 has two regimes, one of them associated with uncongested and one with congested traffic conditions. The density at the state 1 is, according to the inverse of the fundamental diagram, $\rho_{j}^{1}=$ $\rho_{j}\left(Q_{j}^{1} \mid\right.$ uncongested) (if we assume that $\left.Q_{j}^{1}<C_{j}\right)$ and the speed $V_{j}^{1}=Q_{j}^{1} / \rho_{j}^{1}$, while the state 2 is associated with a flow $Q_{j}^{2}, \quad$ a density $\rho_{j}^{2}=\rho^{j}\left(Q_{j}^{2} \mid\right.$ congested $)$ and a speed $V_{j}^{2}=Q_{j}^{2} / \rho_{j}^{2}$. The transition between the two states is propagating through the link $j$ with a speed of

$$
w^{1 \longrightarrow 2}=\frac{Q_{j}^{2}-Q_{j}^{1}}{\rho_{j}^{2}-\rho_{j}^{1}} .
$$

The time discretisation of the CTM was also eliminated by the event-based solution algorithm (eLTM), proposed by Raadsen et al. [51]. Instead of calculating the link boundary conditions at each time step, the algorithm calculates them only for specific discrete events when a change of the sate occurs. An event is triggered when a backward moving shock wave hits the upstream end of a link, when a forward moving shock wave hits the downstream end of a link, or when two shock waves meet each other.

In practice, nodes may be more complicated than the one illustrated in Figure 8, having several incoming and outgoing links. Therefore, a possible downstream supply reduction should be distributed among the incoming links, restricting their outflow accordingly. Node models perform this task by 


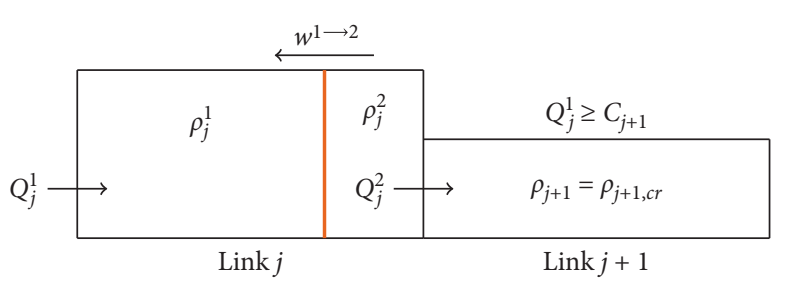

(a)

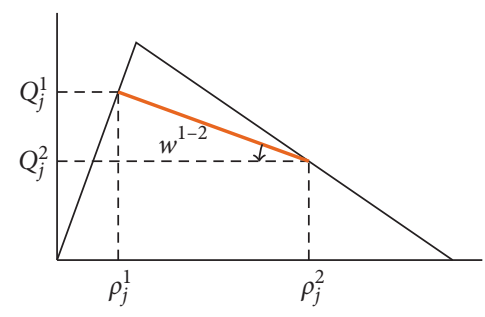

(b)

FIGURE 8: Backward moving shock wave created by reduced capacity on link $j+1$.

maximising the total node flow and ensuring consistency between demand and supply constraints imposed by the incoming and outgoing links. From an emission estimation perspective, node models may be equally important to the link models as they can influence the propagation of congestion. The eLTM link model is coupled with the node model suggested by Tampère et al. [52].

The queuing phase of STAQ adopts an event-based solution algorithm, similar to eLTM. Starting from the sending and receiving flows computed in the squeezing phase, the node model calculates the actual transition rates and determines inflow and outflow rates for each link. Then, a shock wave is generated from any possible difference among such inflows and outflows, as a boundary between the two different traffic states. Therefore, the first events are triggered at the starting time of the algorithm, while the propagation velocity of each shock wave reveals the time for the next candidate events, namely, the expected arrival time of each shock wave to the upstream node of a link. Thus, horizontal queues are built up in front of the bottlenecks which may also spillback to several upstream links. This loading process ends when all traffic demand has reached its destination.

The network loading submodel of STAQ results into link travel times, derived from the cumulative inflow and outflow curves as it is described in the simplified wave theory of Newell [48]. A route-choice submodel, then, computes new path demand shares which are inputs to the squeezing phase, and the procedure is repeated until some convergence criterion is satisfied. Brederode et al. [44] demonstrated that STAQ can improve the modelling accuracy in congested networks compared to STA, without losing in robustness and accountability. Additionally, STAQ keeps the input and computational requirements at lower levels compared to DTA models.

Nevertheless, STAQ considers link interactions, and therefore uniqueness of the solutions is not guaranteed, leading to undesired effects when evaluating different policy scenarios. For this reason, in this study, we solely use the network loading submodel as a postprocess and only for emission estimation purposes. The route choice is exogenous to the model and relies on the traditional VDF, satisfying the uniqueness conditions.

The suggested quasidynamic loading postprocessing approach is based on the first order LWR model similar to the STAQ loading submodel. For the static relationship between flow and density, we use a hyperbolic linear fundamental diagram. Although the transition between two traffic states is not described in detail and may be unrealistic in the LWR model, shock-wave positions are accurately described [39]. For the needs of this study, the latter is more important, since HBEFA emission factors are functions of speed, and the acceleration variations are implicitly modelled, being included in the associated driving cycle. A more complicated emission model, sensitive to deceleration and acceleration, would require a better description of the state's transitions, for instance, by a second-order traffic flow model. Consequently, considering the nature of HBEFA, we use a first-order model, assuming that infinite acceleration can happen instantaneously at the transitions as that does not affect the emission estimates.

4.2. Determination of Emission Factors Based on Queuing Information. Figure 9 illustrates all the possible event types considered in the event-based loading algorithm for a typical network link, $a \in \mathscr{A}$. The events are associated with the transition between four different traffic states, and the propagation speed of those transitions is based on the fundamental diagram of Figure 9. Figure 10 illustrates the cumulative inflow, $\widetilde{U}(t)$, and outflow, $\widetilde{V}(t)$, curves for the boundaries of link $a$, while Figure 11 shows the evolution of the shock waves in the space-time domain. At event time $\varepsilon^{1}$, a backward moving shock wave with speed $w_{a}^{1 \longrightarrow 2}$ hits the downstream end of link $a, a_{L}$, while a faster backward shock wave with speed $w_{a}^{2-3}$ hits the same point at time $\varepsilon^{2}$. The second wave will finally meet the first one, and a new event will occur at time $\varepsilon^{3}$. The new wave that will be formed from their merging will then meet a forward moving wave at $\varepsilon^{5}$. The latter had previously hit the upstream end, $a_{0}$, at $\varepsilon^{4}$. Finally, at time $\varepsilon^{6}$, a new forward wave will reach the downstream end $a_{L}$.

The main output of the event-based loading algorithms described in Bliemer et al. [16] and Brederode et al. [44] is average link travel times computed from the cumulative inflow and outflow curves. Besides the travel times, cumulative flow curves can provide some additional information on inflow and outflow rates and spatial average link densities. Nevertheless, such densities are spatially averaged and regard the entire link. There is no indication as to which part of a link is congested and what are the queues' length. Considering the location-specific effects of emissions, 


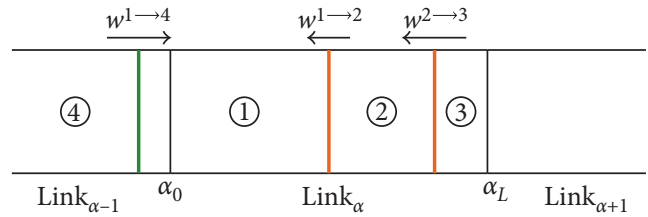

(a)

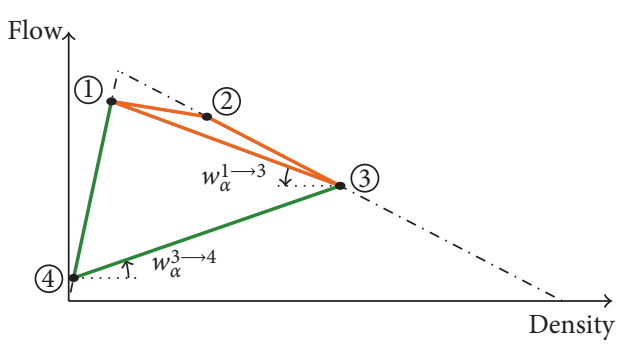

(b)

FIGURE 9: Spatiotemporal evolution of shock waves and cumulative flows.

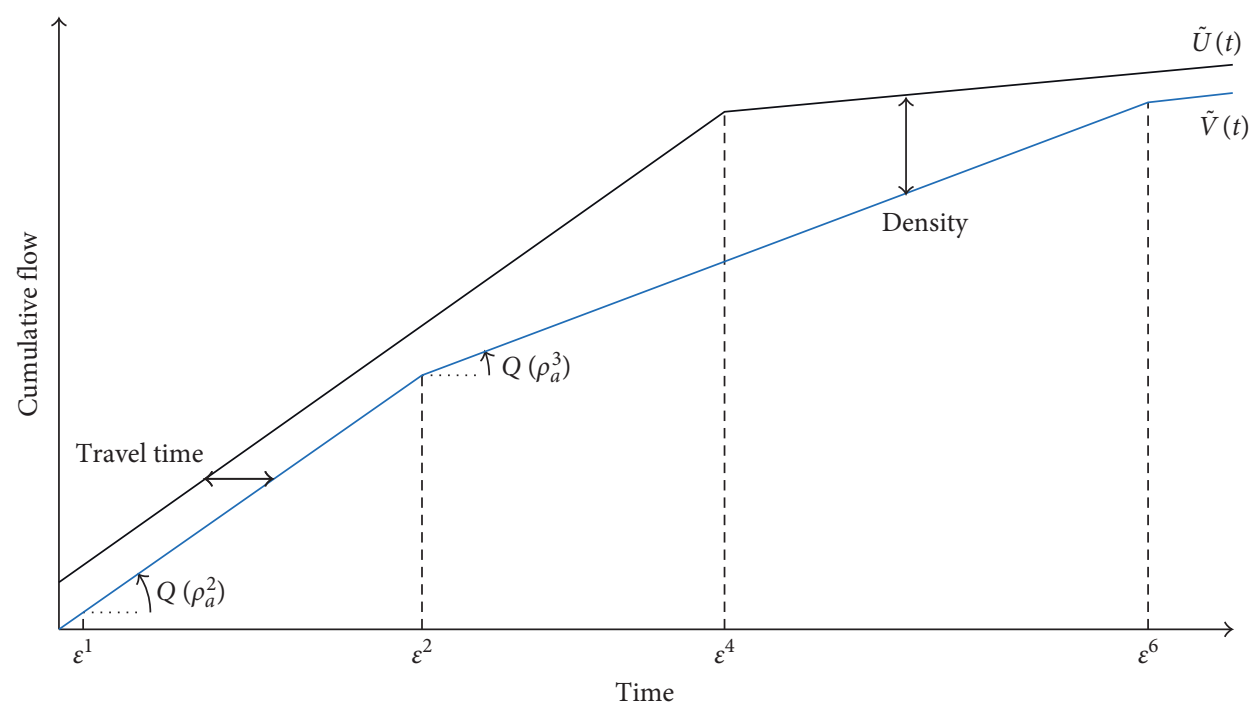

Figure 10: Cumulative inflow and outflow curves.

spatially averaged link speeds may be insufficient for estimating emission at long links.

In our study, to make the model outputs suitable for emission estimation, we employ information coming from the evolution of the shock waves in the space-time domain (Figure 11), instead of using the cumulative flow curves (Figure 10). By applying the event-based loading algorithm of STAQ, it is also possible to keep track of the spatial position of the shock wave whenever an event occurs. From the timedistance plot, we can notice that the shock waves divide a link into different smaller areas, each one of them associated with different traffic state, $[\rho, Q(\rho), V(\rho)]$. Particularly, in the case of Figure 11, the link $a$ is divided into four areas. Each area is related to a specific density $\rho$, flow $Q(\rho)$, as well as to a specific average speed $V(\rho)$ and emission factor $\bar{g}^{p}[V(\rho)]$. Hence, the crucial average link speed, used for the emission estimation, acquires spatial and temporal variability.

Let $\mathscr{M}=\{1,2, \ldots, N-1\}$, where $N$ is the total number of events during the analysis period. Every link of the network, $a \in \mathscr{A}$, at each step $n \in \mathscr{M}$, is divided into $P_{a}^{n}$ parts depending on the number of shock waves, $W_{p}^{n},\left(P_{a}^{n}=W_{p}^{n}+1\right)$ traversing the link at time of the nth event, $\varepsilon^{n}$. Let $\mathscr{P}_{a}^{n}=\left\{1,2, \ldots, P_{a}^{n}\right\}$ and $\mathscr{W}_{a}^{n}=\left\{1,2, \ldots, W_{a}^{n}\right\}$. The algorithm keeps track of the position, namely, the distance from the downstream end of the link, $h_{a, j}^{n}$, and the shock-wave speed, $w_{a, j}^{n}$, of every shock wave, $j \in \mathscr{W}_{a}^{i}$, as well as of the traffic conditions, $\rho_{a, i}^{n}, Q_{a, i}^{n}$, and
$V_{a, i}^{n}$, for each part of the link, $i \in \mathscr{P}_{a}^{n}$. Since in steady-state conditions, shock-wave speeds are constant along the characteristic curves, the transitions between the different traffic states are linear in the space-time domain, and they can be expressed through linear equations of the form $y_{1}=m \cdot y_{2}+b$. The slope $m$ is by definition equal to the shock-wave speed $w_{a, j}^{n}, j \in \mathscr{W}_{a}^{i}$, while the constant $b$ equals to $h_{a, j}^{n}-w_{a, j}^{n} \varepsilon^{n}, j \in \mathscr{W}_{a}^{i}$. Hence, the area in the space-time domain, $R_{a, i}^{n}$, of each part $i \in \mathscr{P}_{a}^{n}$, which is defined between two shock waves and two time steps, can be computed as

$$
\begin{aligned}
R_{a, 1}^{n}= & \int_{\varepsilon^{n}}^{\varepsilon^{n+1}}\left(w_{a, 1}^{n} \omega+h_{a, 1}^{n}-w_{a, 1}^{n} \varepsilon^{n}\right) \mathrm{d} \omega, \\
R_{a, i}^{n}= & \int_{\varepsilon^{n}}^{\varepsilon^{n+1}}\left(w_{a, i}^{n} \omega+h_{a, i}^{n}-w_{a, i}^{n} \varepsilon^{n}\right) \mathrm{d} \omega \\
& -\int_{\varepsilon^{n}}^{\varepsilon^{n+1}}\left(w_{a, i-1}^{n} \omega+h_{a, i-1}^{n}-w_{a, i-1}^{n} \varepsilon^{n}\right) \mathrm{d} \omega, \\
R_{a, P_{a}^{n}}^{n}= & \left(a_{L}-a_{0}\right)\left(\varepsilon^{n+1}-\varepsilon^{n}\right) \quad \forall \mathscr{P}_{a}^{n} /\left\{1, P_{a}^{n}\right\}, \\
& -\int_{\varepsilon^{n}}^{\varepsilon^{n+1}}\left(w_{a, W_{a}^{n}}^{n} \omega+h_{a, W_{a}^{n}}^{n}-w_{a, W_{a}^{n}}^{n} \varepsilon^{n}\right) \mathrm{d} \omega .
\end{aligned}
$$




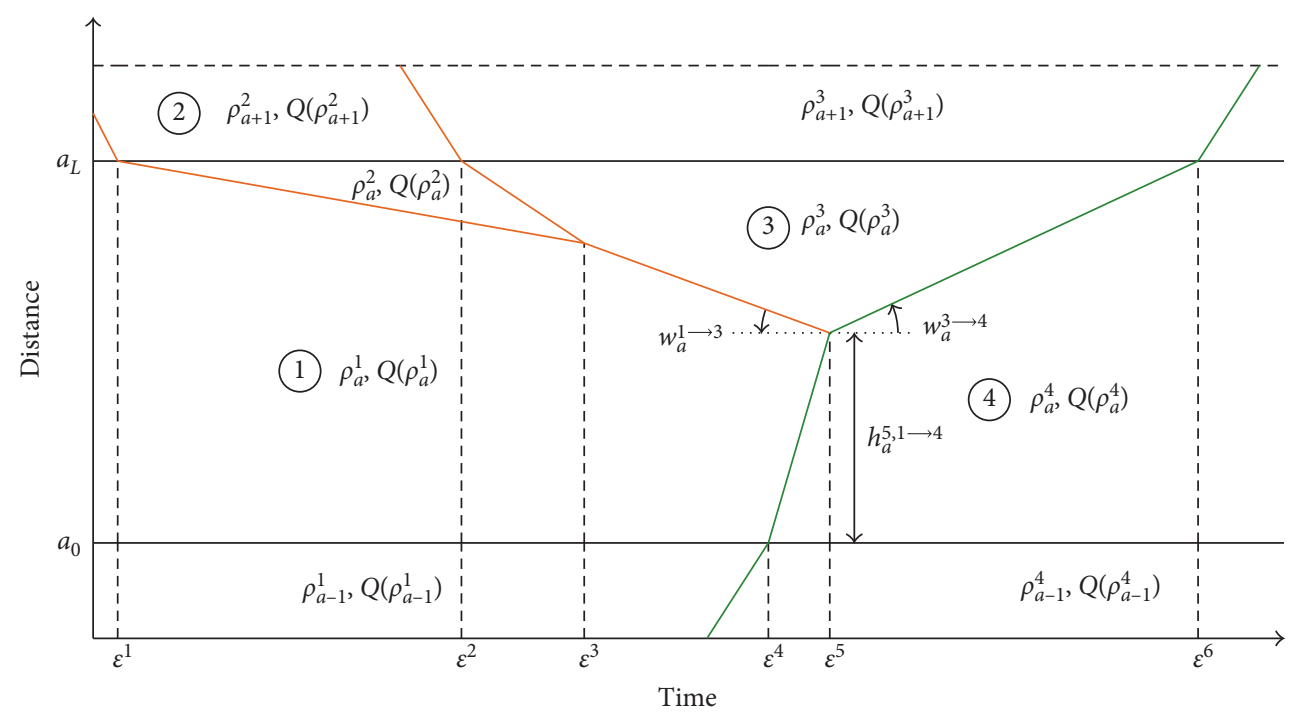

FIGURE 11: Spatiotemporal evolution of shock waves.

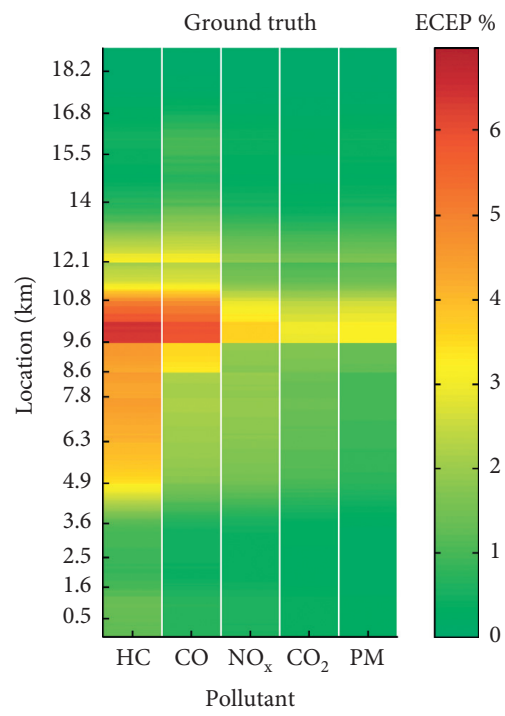

(a)

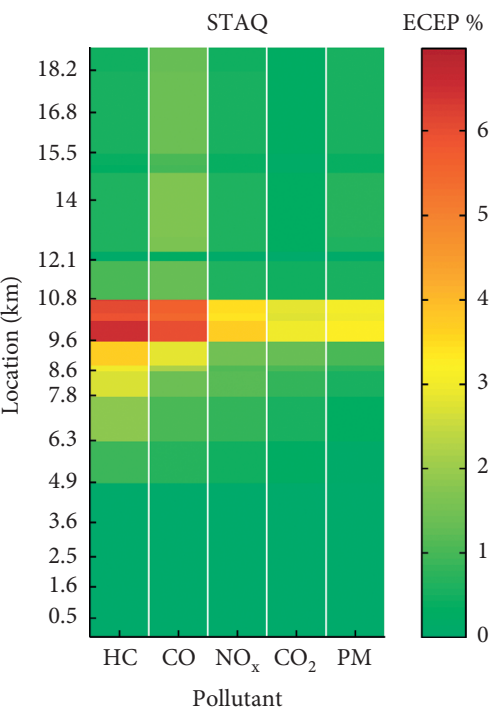

(b)

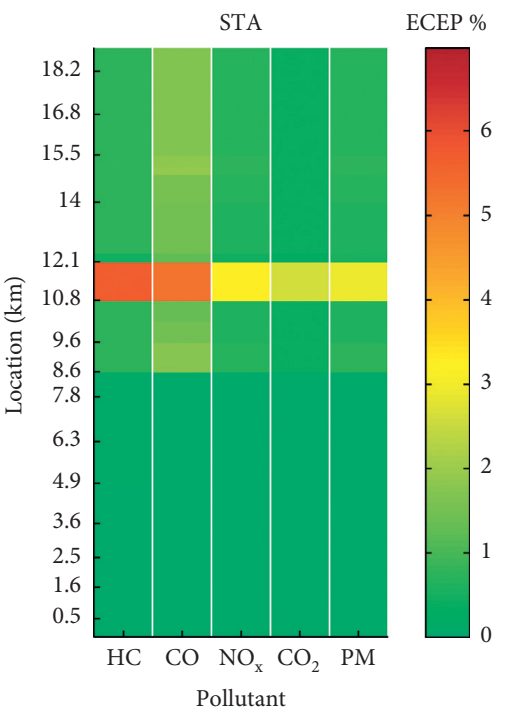

(c)

FIgUre 12: Average excess due to congestion emissions (ECEP) for (a) the ground truth, (b) static loading with queuing, and (c) static loading.

The area multiplied by the flow rate at step $n$, $Q_{a, i}^{n}, i \in \mathscr{P}_{a}^{n}$, gives the vehicle kilometres travelled for the specific state. Finally, for each link $a$, the grams of pollutant, $p$, emitted can be estimated as

$$
\widehat{E}_{a}^{p}=\sum_{n \in \mathscr{M}} \sum_{i \in \mathscr{P}_{a}^{n}} R_{a, i}^{n} \cdot Q_{a, i}^{n} \cdot \bar{g}_{a}^{p}\left(V_{a, i}^{n}\right) .
$$

A more detailed description of the algorithm can be found in Tsanakas [53].

4.3. STAQResults for the Case Study Segment. Our hypothesis is that the application of quasidynamic loading as a postprocessing technique could potentially reduce the emissions estimation errors introduced by static models. To test our hypothesis, we applied the suggested postprocessing approach for the same Stockholm's test site. The results are illustrated in Figure 12, expressed in ECEP terms, with the STAQ ECEP being computed as

$\operatorname{ECEP}_{a}^{p}=\left(\sum_{n \in \mathscr{M}} \sum_{i \in \mathscr{P}_{a}^{n}} \widehat{u}_{a, i}^{n} \bar{g}_{a}^{p}\left(V_{a, i}^{n}\right)-\bar{g}_{a}^{p}\left(V_{a}^{0}\right)\right) \frac{100}{\bar{g}_{a}^{p}\left(V_{a}^{0}\right)}$,

where $V_{a}^{0}$ is the free flow speed of link $a$ and $\widehat{u}_{a, i}^{n}$ is a weighting factor that reflects the spatiotemporal variability of the emission factors by relating the area of part $i, i \in \mathscr{P}_{a}^{n}$, with the total space-time area of link $a$,

$$
\widehat{u}_{a, i}^{n}=\frac{R_{a, i}^{n}}{\left(a_{L}-a_{0}\right) T}
$$

where $T$ is the total duration of the analysis period. 
As it is depicted in Figure 12(b), the excess emissions are estimated upstream the bottleneck while the queues are propagated backwards, spilling back to several links. Therefore, in contrast to the static case (Figure 12(c)), the higher excess emissions are located almost at the same positions as the ground truth. Figure 12 demonstrates that postprocessing the static assignment outputs can actually lead to a more accurate spatial distribution of emissions for our case study motorway section. It is clear that the errors are now significantly reduced, especially for the links upstream the bottleneck. In terms of total segment emissions, we also notice an improvement since the differences with the ground truth now range from $0.23 \%$ to $1.05 \%$ depending on the pollutant.

\section{Conclusions and Future Research}

For large-scale emission estimation analyses, the required traffic conditions are usually obtained by static models. In this paper, we show that their simplified way of representing traffic can lead to considerable emission estimation errors. Aggregated over time and space, average traffic conditions tend to underestimate the emissions, especially in the case of a discrete emission model such as the HBEFA. Nevertheless, we demonstrate that using a continuous emission model, the spatiotemporal resolution problem can be satisfactorily moderated.

In addition, the inability of static models to describe dynamic traffic flow phenomena can also cause significant emission estimation errors. These errors are mainly associated with the inaccurate modelling of congestion's location which may lead to an unreliable spatial distribution of emissions. Even though STA results may be sufficient for travel timebased analyses, in the case of emission estimation, we support that STA outputs need supplementary postprocessing. Since the problem is identified at the network loading, we suggest the use of a quasidynamic loading model for postprocessing the STA results. Our results show that this approach could lead to more accurate spatial allocation of emissions.

For aggregated applications of emission modelling, such as annual emission inventories, the spatial distribution may not be so important. Other factors, e.g., vehicles fleet composition, can be more influential. Therefore, in such cases, STA without postprocessing could be adequate to generate inputs for the emission model. However, for applications such as dispersion and exposure modelling, where spatial variations of emission rates are vital, postprocessing could offer considerable improvements.

It would be interesting for the future to estimate emissions for a large-scale network using the quasidynamic loading and evaluate the effect for applications like air quality modelling. Future studies could also investigate the association between the spatial distribution of emissions due to postprocessing with the damage cost of a pollutant and evaluate the use of quasidynamic loading in economic appraisals.

We should note here that in this study, we consider spatially and temporally constant vehicle fleet mixture. In addition, we do not take into account the heavy-duty vehicles, which constitute almost $5 \%$ of the total fleet. The inclusion of heavy-duty vehicles and a varied in time and
TABLE 2: The considered vehicle composition for passenger cars (PC) (petrol (P) and diesel (D)) according to the European standards for light-duty vehicles.

\begin{tabular}{lc}
\hline Vehicle class & \% of the total fleet \\
\hline PC-P-Euro-0 & 0.93 \\
PC-P-Euro-1 & 1.14 \\
PC-P-Euro-2 & 7.63 \\
PC-P-Euro-3 & 4.25 \\
PC-P-Euro-4 & 24.25 \\
PC-P-Euro-5 & 9.09 \\
PC-P-Euro-6 & 0.25 \\
PC-alternative fuel & 14.55 \\
PC-D-Euro-0 & 0.03 \\
PC-D-Euro-1 & 0.06 \\
PC-D-Euro-2 & 0.37 \\
PC-D-Euro-3 & 1.11 \\
PC-D-Euro-4 & 12.35 \\
PC-D-Euro-5 & 23.00 \\
PC-D-Euro-6 & 0.99 \\
\hline
\end{tabular}

TABLE 3: The HBEFA emission factors used for this study in grams of pollutant emitted per kilometre for five pollutants (HC, CO, $\mathrm{NO}_{\mathrm{x}}, \mathrm{CO}_{2}$, and $\mathrm{PM}_{10}$ ), four traffic situations (Free flow, Heavy, Saturated, and Stop and go), and for two road types, urban motorway with a speed limit of $70 \mathrm{~km} / \mathrm{h}$ (URB/MW/70) and urban motorway with a speed limit of $90 \mathrm{~km} / \mathrm{h}$ (URB/MW/90).

\begin{tabular}{lccc}
\hline Pollutant & Traffic situation & URB/MW/70 & URB/MW/90 \\
\hline \multirow{4}{*}{$\mathrm{HC}$} & Free flow & 0.016 & 0.016 \\
& Heavy & 0.022 & 0.016 \\
& Saturated & 0.022 & 0.021 \\
& Stop and go & 0.073 & 0.057 \\
$\mathrm{CO}$ & Free flow & 0.167 & 0.278 \\
& Heavy & 0.337 & 0.219 \\
& Saturated & 0.261 & 0.336 \\
& Stop and go & 0.711 & 0.622 \\
\hline \multirow{4}{*}{$\mathrm{NO}_{\mathrm{x}}$} & Free flow & 0.174 & 0.194 \\
& Heavy & 0.240 & 0.189 \\
& Saturated & 0.223 & 0.220 \\
& Stop and go & 0.524 & 0.406 \\
$\mathrm{CO}_{2}$ & Free flow & 109.95 & 127.98 \\
& Heavy & 132.88 & 121.50 \\
& Saturated & 133.30 & 132.69 \\
& Stop and go & 290.80 & 229.54 \\
\hline \multirow{3}{*}{$\mathrm{PM}_{10}$} & Free flow & 0.0027 & 0.0037 \\
& Heavy & 0.0038 & 0.0032 \\
& Saturated & 0.0034 & 0.0037 \\
& Stop and go & 0.0075 & 0.0058 \\
\hline
\end{tabular}

space vehicle fleet could affect our results, without influencing our main findings related to the location of the excess emissions.

\section{Appendix}

\section{A. Vehicle Fleet Composition in Sweden}

The vehicle composition for passenger cars is given in Table 2. 


\section{B. HBEFA Emission Factors}

The HBEFA emission factors used for this study is given in Table 3.

\section{Data Availability}

The traffic data used to support the findings of this study are available from the corresponding author upon request.

\section{Conflicts of Interest}

The authors declare that there are no conflicts of interest.

\section{Acknowledgments}

This work was supported by the Swedish Energy Agency (grant no. 38921-1).

\section{References}

[1] EEA, “Air quality in Europe-2016," Technical report 28/201, European Environment Agency, Copenhagen, Denmark, 2016.

[2] Y. Sheffi, Urban Transportation Network: Equilibrium Analysis with Mathematical Programming Methods, Prentice-Hall, Upper Saddle River, NJ, USA, 1985.

[3] M. C. J. Bliemer, M. P. H. Raadsen, L. J. N. Brederode, M. G. H. Bell, L. J. J. Wismans, and M. J. Smith, "Genetics of traffic assignment models for strategic transport planning," Transport Reviews, vol. 37, no. 1, pp. 56-78, 2017.

[4] S. Bai, Y. Nie, and D. A. Niemeier, "The impact of speed postprocessing methods on regional mobile emissions estimation," Transportation Research Part D: Transport and Environment, vol. 12, no. 5, pp. 307-324, 2007.

[5] M. Bliemer, M. Raadsen, E. d. Romph, and E.-S. Smits, "Requirements for traffic assignment models for strategic transport planning: a critical assessment," in Proceedings of the 2013 Australasian Transport Research Forum, Brisbane, Australia, October 2013.

[6] Flügel, S. and Flötteröd, G. (2015). Traffic Assignment for Strategic Urban Transport Model Systems.

[7] Y. Wang, W. Y. Szeto, K. Han, and T. L. Friesz, "Dynamic traffic assignment: a review of the methodological advances for environmentally sustainable road transportation applications," Transportation Research Part B: Methodological, vol. 111, pp. 370-394, 2018.

[8] L. Wismans, E. Van Berkum, and M. Bliemer, "Modelling externalities using dynamic traffic assignment models: a review," Transport Reviews, vol. 31, no. 4, pp. 521-545, 2011.

[9] J. Eliasson, "A cost-benefit analysis of the stockholm congestion charging system," Transportation Research Part A: Policy and Practice, vol. 43, no. 4, pp. 468-480, 2009.

[10] H. M. A. Aziz and S. V. Ukkusuri, "Integration of environmental objectives in a system optimal dynamic traffic assignment model," Computer-Aided Civil and Infrastructure Engineering, vol. 27, no. 7, pp. 494-511, 2012.

[11] R. Smit, M. Poelman, and J. Schrijver, "Improved road traffic emission inventories by adding mean speed distributions," Atmospheric Environment, vol. 42, no. 5, pp. 916-926, 2008.

[12] A. Aguilera and M. Lebacque, "Dynamic estimation of traffic emissions in metropolitan road networks," in Proceedings of the 12th WCTR, Lisbon, Portugal, July 2010.
[13] R. Borge, I. de Miguel, D. de la Paz, J. Lumbreras, J. Pérez, and E. Rodríguez, "Comparison of road traffic emission models in Madrid (Spain)," Atmospheric Environment, vol. 62, pp. 461-471, 2012.

[14] X. Zhou, S. Tanvir, H. Lei et al., "Integrating a simplified emission estimation model and mesoscopic dynamic traffic simulator to efficiently evaluate emission impacts of traffic management strategies," Transportation Research Part D: Transport and Environment, vol. 37, pp. 123-136, 2015.

[15] L. Wismans, R. M. M. van de Brink, L. J. N. Brederode, K. J. Zantema, and E. C. van Berkum, "Comparison of estimation of emissions based on static and dynamic traffic assignment," in Proceedings of the 93rd Annual Meeting of the Trasportation Research Board, Washington, DC, USA, 2013.

[16] M. C. J. Bliemer, L. Brederode, L. J. J. Wismans, and E. S. Smits, "Quasi-dynamic traffic assignment: static traffic assignment with queuing and spillback," in Proceedings of the 94th Annual Meeting of the Trasportation Research Board, Washington, DC, USA, January 2015.

[17] M. Bundschuh, P. Vortisch, T. Van Vuuren, and M. McDonald, "Modelling queues in static traffic assignment," in Proceedings of the 2006 European Transport Conference, Strasbourg, France, September 2006.

[18] N. Tsanakas, J. Ekström, and J. Olstam, "Estimating emissions from static traffic models-problems and solutions," in Proceedings of the 2017 Swedish Transportation Research Conference-Conference Program and Book of Abstracts, Linköping, Sweden, October 2017.

[19] N. Tsanakas, J. Ekström, and J. Olstam, "Reduction of errors when estimating emissions based on static traffic model outputs," Transportation Research Procedia, vol. 22, pp. 440449, 2017.

[20] V. Franco, M. Kousoulidou, M. Muntean, L. Ntziachristos, S. Hausberger, and P. Dilara, "Road vehicle emission factors development: a review," Atmospheric Environment, vol. 70, pp. 84-97, 2013.

[21] V. Franco, G. Fontaras, and P. Dilara, "Towards improved vehicle emissions estimation in Europe," Procedia-Social and Behavioral Sciences, vol. 48, pp. 1304-1313, 2012.

[22] M. Keller, "Handbook of emission factors for road transport (HBEFA) 3.1. Technical report, quick reference," Technical report, INFRAS, Zürich, Switzerland, 2010.

[23] L. Ntziachristos, D. Gkatzoflias, C. Kouridis, and Z. Samaras, "COPERT: a European road transport emission inventory model," in Information Technologies in Environmental Engineering, pp. 491-504, Springer, Berlin, Germany, 2009.

[24] EEA, "EMEP/EEA air pollutant emission inventory guidebook 2016," EEA report No 21/2016, European Environment Agency, Copenhagen, Denmark, 2016.

[25] J. G. Wardrop and J. I. Whitehead, "Correspondence. Some theoretical aspects of road traffic research," Proceedings of the Institution of Civil Engineers, vol. 1, no. 5, pp. 767-768, 1952.

[26] M. J. Beckmann, C. B. McGuire, and C. B. Winsten, Studies in the Economics of Transportation, Yale University Press, New Haven, CT, USA, 1956.

[27] Trafikverket, Handbok för Vägtrafikens Luftföroreningar. Kapitel 6, Emissionsdatabaser, 2012.

[28] M. Barth, G. Scora, and T. Younglove, "Estimating emissions and fuel consumption for different levels of freeway congestion," Transportation Research Record: Journal of the Transportation Research Board, vol. 1664, no. 1, pp. 47-57, 1999.

[29] S. Bharadwaj, S. Ballare, Rohit, and M. K. Chandel, "Impact of congestion on greenhouse gas emissions for road transport in 
Mumbai metropolitan region," Transportation Research Procedia, vol. 25, pp. 3538-3551, 2017.

[30] K. Zhang, S. Batterman, and F. Dion, "Vehicle emissions in congestion: comparison of work zone, rush hour and freeflow conditions," Atmospheric Environment, vol. 45, no. 11, pp. 1929-1939, 2011.

[31] S. E. Nicholson, "A pollution model for street-level air," Atmospheric Environment (1967), vol. 9, no. 1, pp. 19-31, 1975.

[32] S. Vardoulakis, B. E. A. Fisher, K. Pericleous, and N. Gonzalez-Flesca, "Modelling air quality in street canyons: a review," Atmospheric Environment, vol. 37, no. 2, pp. 155-182, 2003.

[33] E. Negrenti, "The "corrected average speed" approach in enea's tee model: an innovative solution for the evaluation of the energetic and environmental impacts of urban transport policies," Science of the Total Environment, vol. 235, no. 1-3, pp. 411-413, 1999.

[34] K. S. Nesamani, L. Chu, M. G. McNally, and R. Jayakrishnan, "Estimation of vehicular emissions by capturing traffic variations," Atmospheric Environment, vol. 41, no. 14, pp. 2996-3008, 2007.

[35] B. Y. Ryu, H. J. Jung, and S. H. Bae, "Development of a corrected average speed model for calculating carbon dioxide emissions per link unit on urban roads," Transportation Research Part D: Transport and Environment, vol. 34, pp. 245-254, 2015.

[36] R. Akcelik, "Travel time functions for transport planning purposes: Davidson's function, its time dependent form and alternative travel time function," Australian Road Research, vol. 21, no. 3, 1991.

[37] R. Dowling and A. Skabardonis, "Improving average travel speeds estimated by planning models," Transportation Research Record, no. 1366, pp. 68-74, 1993.

[38] M. Treiber and D. Helbing, "Reconstructing the spatiotemporal traffic dynamics from stationary detector data," Cooper@TiveTr@Nsport@TionDyn@Mics, vol. 1, no.3, pp. 3.1-3.24, 2002.

[39] M. Treiber and A. Kesting, Traffic Flow Dynamics: Data, Models and Simulation, Springer-Verlag, Berlin, Germany, 2013.

[40] D. B. Work, S. Blandin, O.-P. Tossavainen, B. Piccoli, and A. M. Bayen, "A traffic model for velocity data assimilation," Applied Mathematics Research eXpress, vol. 2010, no. 1, pp. 1-35, 2010.

[41] N. Tsanakas, J. Ekström, and J. Olstam, "Emission estimation based on cross-sectional traffic data," in Proceedings of the 22nd International Transport and Air Pollution Conference, Zürich, Switzerland, September 2017.

[42] U. Björketun and A. Carlsson, "Trafikvariation över dygnet," Technical report, VTI, Linköping, Sweden, 2005.

[43] M. C. J. Bliemer, M. P. H. Raadsen, E.-S. Smits, B. Zhou, and M. G. H. Bell, "Quasi-dynamic traffic assignment with residual point queues incorporating a first order node model," Transportation Research Part B: Methodological, vol. 68, pp. 363-384, 2014.

[44] L. Brederode, A. Pel, L. Wismans, E. de Romph, and S. Hoogendoorn, "Static traffic assignment with queuing: model properties and applications," Transportmetrica A: Transport Science, vol. 15, no. 2, pp. 179-214, 2019.

[45] M. J. Lighthill and G. B. Whitham, "On kinematic waves. II. A theory of traffic flow on long crowded roads," Proceedings of the Royal Society of London A: Mathematical, Physical and Engineering Sciences, vol. 229, no. 1178, pp. 317-345, 1955.
[46] P. I. Richards, "Shock waves on the highway," Operations Research, vol. 4, no. 1, pp. 42-51, 1956.

[47] W. Leutzbach, Introduction to the Theory of Traffic Flow, vol. 47, Springer, Berlin, Germany, 1988.

[48] G. F. Newell, "A simplified theory of kinematic waves in highway traffic, part II: queueing at freeway bottlenecks," Transportation Research Part B: Methodological, vol. 27, no. 4, pp. 289-303, 1993.

[49] C. F. Daganzo, "The cell transmission model: a dynamic representation of highway traffic consistent with the hydrodynamic theory," Transportation Research Part B: Methodological, vol. 28, no. 4, pp. 269-287, 1994.

[50] I. Yperman, the link transmission model for dynamic network loading., Ph.D. thesis, Katholieke Universiteit Leuven, Leuven, Belgium, 2007.

[51] M. P. H. Raadsen, M. C. J. Bliemer, and M. G. H. Bell, “An efficient and exact event-based algorithm for solving simplified first order dynamic network loading problems in continuous time," Transportation Research Part B: Methodological, vol. 92, pp. 191-210, 2016.

[52] C. M. J. Tampère, R. Corthout, D. Cattrysse, and L. H. Immers, "A generic class of first order node models for dynamic macroscopic simulation of traffic flows," Transportation Research Part B: Methodological, vol. 45, no. 1, pp. 289-309, 2011.

[53] N. Tsanakas, "Emission estimation based on traffic models and measurements," Licentiate thesis, Linköping University Electronic Press, Linköping, Sweden, 2019. 